\title{
Synthesis of trisaccharide methyl glycosides related to fragments of the capsular polysaccharide of Streptococcus pneumoniae type $18 \mathrm{C}$
}

\author{
Augusta M. P. van Steijn, Johannis P. Kamerling*, and Johannes F. G. Vliegenthart \\ Bijvoet Center, Department of Bio-Organic Chemistry, Utrecht University, P.O. Box 80.075, NL-3508 TB \\ Utrecht (The Netherlands)
}

(Received June 15th, 1991; accepted August 13th, 1991)

\section{ABSTRACT}

The synthesis is reported of methyl 3-O-(4-O- $\beta$-D-galactopyranosyl- $\alpha$-D-glucopyranosyl)- $\alpha$-L-rhamnopyranoside (1), methyl $2-O-\alpha$-D-glucopyranosyl-4- $O-\beta$-D-glucopyranosyl- $\beta$-D-galactopyranoside (3), me-

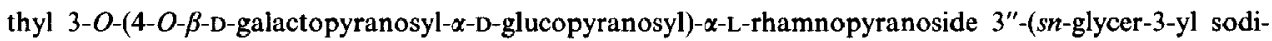
um phosphate) (2), and methyl 2-O- $\alpha$-D-glucopyranosyl-4- $O-\beta$-D-glucopyranosyl- $\beta$-D-galactopyranoside 3(sn-glycer-3-yl sodium phosphate) (4), which are trisaccharide methyl glycosides related to fragments of the capsular polysaccharide of Streptococcus pneumoniae type 18C $(\{\rightarrow 4)-\beta$-D-Glc $p-(1 \rightarrow 4)-[\alpha-\mathrm{D}-\mathrm{Glc} p-(1 \rightarrow 2)]-$ [Glycerol-( $(1-\mathrm{P} \rightarrow 3)]-\beta$-D-Galp-( $\left.(1 \rightarrow 4)-\alpha-\mathrm{D}-\mathrm{Gl} c p-(1 \rightarrow 3)-\alpha-\mathrm{L}-\mathrm{Rhap}-(1 \rightarrow\}_{n}\right)$.

Ethyl 4- $O$-acetyl-2,3,6-tri- $O$-benzyl-1-thio- $\beta$-D-glucopyranoside (10) was coupled with benzyl 2,4di- $O$-benzyl- $\alpha$-L-rhamnopyranoside (6). Deacetylation of the product, followed by condensation with $2,4,6-$ tri- $O$-acetyl-3-O-allyl- $\alpha$-D-galactopyranosyl trichloroacetimidate (18), gave benzyl 2,4-di- $O$-benzyl-3-O[2,3,6-tri- $O$-benzyl-4- $O$-(2,4,6-tri- $O$-acetyl-3- $O$-allyl- $\beta$-D-galactopyranosyl)- $\alpha$-D-glucopyranosyl]- $\alpha$-Lrhamnopyranoside (19). Acetolysis of 19, followed by methylation, deallylation $(\rightarrow \mathbf{2 2})$, and further deprotection afforded 1.

Condensation of methyl 2,4-di- $O$-benzyl-3- $O$-[2,3,6-tri- $O$-benzyl-4- $O$-(2,4,6-tri- $O$-acetyl- $\beta$-D-galactopyranosyl)- $\alpha$-D-glucopyranosyl]- $\alpha$-L-rhamnopyranoside (22) with 1,2-di- $O$-benzyl-sn-glycerol 3-(triethylammonium phosphonate) (24), followed by oxidation and deprotection, yielded 2.

Condensation of ethyl 2,3,4,6-tetra-O-benzyl-1-thio- $\beta$-D-glucopyranoside (27) with methyl 3-Oallyl-4,6- $O$-benzylidene- $\beta$-D-galactopyranoside (28), selective benzylidene ring-opening of the product, coupling with $2,3,4,6$-tetra- $O$-acetyl- $\alpha$-D-glucopyranosyl trichloroacetimidate (31), and deallylation afforded methyl 6-O-benzyl-4- $O$-(2,3,4,6-tetra- $O$-acetyl- $\beta$-D-glucopyranosyl)-2- $O$-(2,3,4,6-tetra- $O$-benzyl- $\alpha$-Dglucopyranosyl)- $\beta$-D-galactopyranoside (33). Deprotection of 33 gave 3 , and condensation of 33 with 24 , followed by oxidation and deprotection, gave 4.

\section{INTRODUCTION}

The current polysaccharide vaccine Pneumovax 23 against such pneumococcal diseases as pneumonia, otitis media, and meningitis contains the capsular polysaccharides isolated from 23 species of Streptococcus pneumoniae. In view of the immunological problems associated with this vaccine, much attention has been paid to the preparation of better alternatives based on polysaccharide or oligosaccharide conjugates,

\footnotetext{
* Author for correspondence.
}

$0008-6215 / 92 \$ 05.00 \quad$ (C) 1992 - Elsevier Science Publishers B.V. All rights reserved. 
having proteins as the carrier ${ }^{1}$. We now report the synthesis of the trisaccharide methyl glycosides 1-4, which are related to fragments of the capsular polysaccharide (5) of $S$. pneumoniae serotype $18 \mathrm{C}$ (ref. 2), one of the constituents of the vaccine ${ }^{3}$.

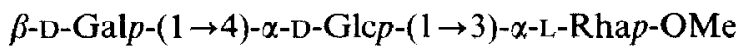

1

$s n$-Glycerol-(3-P $\rightarrow 3)-\beta$-D-Gal $p-(1 \rightarrow 4)-\alpha-\mathrm{D}-\mathrm{Gl} p p-(1 \rightarrow 3)-\alpha$-L-R hap-OMe

2

$\alpha$-D-Glcp-(1 $\rightarrow 2)-[\beta$-D-Glcp-( $(\rightarrow 4)]-\beta$-D-Galp-OMe

3

$\alpha$-D-Glcp-( $(1 \rightarrow 2)-[s n$-Glycerol-(3-P $\rightarrow 3)]-[\beta$-D-Glcp- $(1 \rightarrow 4)]-\beta$-D-Galp-OMe

4

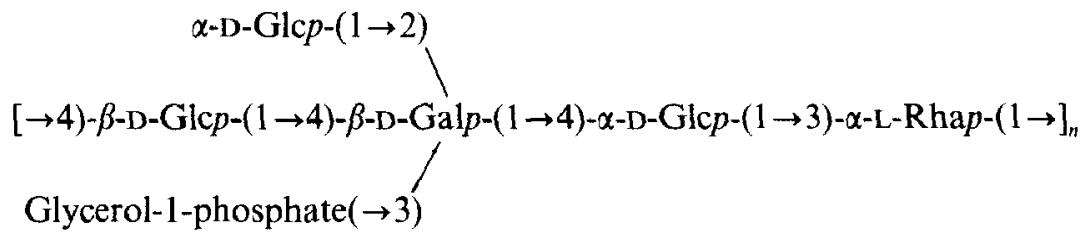

RESULTS AND DISCUSSION

The syntheses of 1 and 2 involved the synthons benzyl 2,4-di- $O$-benzyl- $\alpha-\mathrm{L}-$ rhamnopyranoside ${ }^{4}(6)$, ethyl 4 - $O$-acetyl-2,3,6-tri- $O$-benzyl-1-thio- $\beta$-D-glucopyranoside (10), and 2,4,6-tri- $O$-acetyl-3-O-allyl- $\alpha$-D-galactopyranosyl trichloroacetimidate (18). Benzylidenation of ethyl 1-thio- $\beta$-D-glucopyranoside ${ }^{5}$ with $\alpha, \alpha$-dimethoxytoluene ${ }^{6}$ $\left(\rightarrow 7,69 \%\right.$; lit. $\left.{ }^{5} 47 \%\right)$, followed by benzylation $(\rightarrow 8,81 \%)$, regioselective reductive opening of the 4,6-O-benzylidene ring using the borane-trimethylamine complex and aluminium(III) chloride in tetrahydrofuran ${ }^{7}(\rightarrow 9,73 \%)$, and acetylation afforded 10 . Condensation of 2,3,4,6-tetra- $O$-acetyl- $\alpha$-D-galactopyranosyl trichloroacetimidate ${ }^{8}$ with 4-methoxybenzyl alcohol in dichloromethane, using trimethylsilyl triflate as a catalyst $(\rightarrow \mathbf{1 3}, 73 \%)$, followed by deacetylation $(\rightarrow 14)$, selective allylation with allyl bromide in the presence of tetrabutylammonium iodide $(\rightarrow 15)$, and acetylation gave crystalline 16 (42\% from 13). Removal of the 4-methoxybenzyl group from 16 in the presence of ceric ammonium nitrate ${ }^{10}(\rightarrow \mathbf{1 7}, 81 \%)$ and imidation gave 18 . A synthesis of 

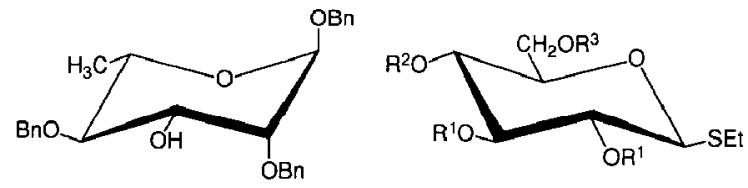

6

$7 \mathrm{R}^{1}=\mathrm{H}, \mathrm{R}^{2}, \mathrm{R}^{3}=\mathrm{CHPh}$

$8 \mathrm{R}^{1}=\mathrm{Bn}, \mathrm{R}^{2}, \mathrm{R}^{3}=\mathrm{CHPh}$

$9 R^{1}=R^{3}=B n, R^{2}=H$

$10 R^{1}=R^{3}=B n, R^{2}=A C$

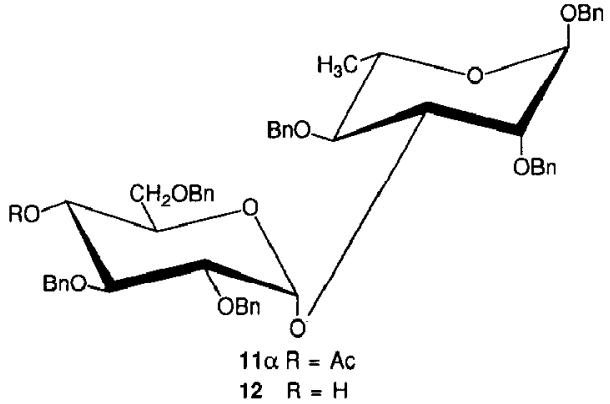

$12 \mathrm{R}=\mathrm{H}$

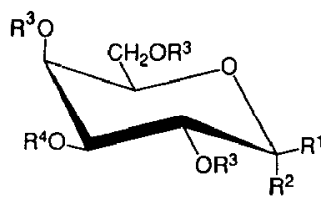

$13 R^{1}=4-M e O B n, R^{2}=H, R^{3}=R^{4}=A C$

$14 R^{1}=4-M e O B n, R^{2}=R^{3}=R^{4}=H$

$15 R^{1}=4-M e O B n, R^{2}=R^{3}=H, R^{4}=$ allyl

$16 R^{1}=4-M e O B n, R^{2}=H, R^{3}=A c, R^{4}=$ allyl

$17 R^{1}, R^{2}=H, O H, R^{3}=A C, R^{4}=$ allyl

$18 \mathrm{R}^{1}=\mathrm{H}, \mathrm{R}^{2}=\mathrm{OCNHCCl}_{3}, \mathrm{R}^{3}=\mathrm{AC}, \mathrm{R}^{4}=$ allyl

17 (and 18) from methyl 2,4,6-tri- $O$-acetyl-3- $O$-allyl- $\beta$-D-galactopyranoside has been described $^{11}$; however, the acetolysis of $\mathrm{MeO}-1$ remained a problem.

Condensation of 10 with 6 in ether, using methyl triflate ${ }^{12}$ as a promoter, gave the disaccharide derivatives $11 \alpha(57 \%)$ and $11 \beta(31 \%)$. Deacetylation of $11 \alpha$ yielded 12 $(98 \%)$. Coupling of 18 with 12 in dry dichloromethane at $-30^{\circ}$, using trimethylsilyl triflate as a catalyst, afforded the trisaccharide derivative $19(73 \%)$.

The synthesis of the methyl $\alpha$-glycoside of 19 , using a non-participating group at C-2 of the rhamnose residue, was studied in order to identify the best conditions for the introduction of a spacer element. Acetolysis of 19 with sulfuric acid in acetic anhydrideacetic acid replaced $\mathrm{BnO}-1$ by $\mathrm{AcO}-1$ and gave 20 (78\%). Replacement of the axial AcO-1 in 20 by an axial MeO-1 $(\rightarrow 21,62 \%)$ was carried out in dichloromethane using methanol with trimethylsilyl triflate as the promoter ${ }^{13}$. Attempts to prepare 21 via the corresponding glycosyl imidates or glycosyl bromide failed. Removal of AcO-1 of 20 with hydrazine acetate, followed by conversion of the resulting trisaccharide derivative into the corresponding glycosyl imidates (1,8-diazabicyclo[5.4.0]undec-7-ene, potassium carbonate) or glycosyl bromide (Vilsmeier-Haack reagent), and condensation with methanol applying different promoters in different solvents afforded always $\alpha, \beta$-mixtures of the methyl glycosides. Deallylation of 21 using palladium(II) chloride ${ }^{14}$ in acetic acid $(\rightarrow 22,75 \%)$, then deacetylation, and debenzylation yielded the target methyl glycoside 1. The ${ }^{1}$ H-n.m.r. data of 1 , obtained by $2 \mathrm{D} \mathrm{COSY}^{15}$ and HOHAHA ${ }^{16}$ measurements, are given in Table I.

For the introduction of the glycerol phosphate group at HO-3 of the galactose moiety of 22, 1,2-di-O-benzyl-sn-glycerol 3-(triethylammonium phosphonate) (24, $23 \%$ ) was prepared by converting 1,2-di-O-benzyl-sn-glycerol ${ }^{17}(23)$ into the corre- 


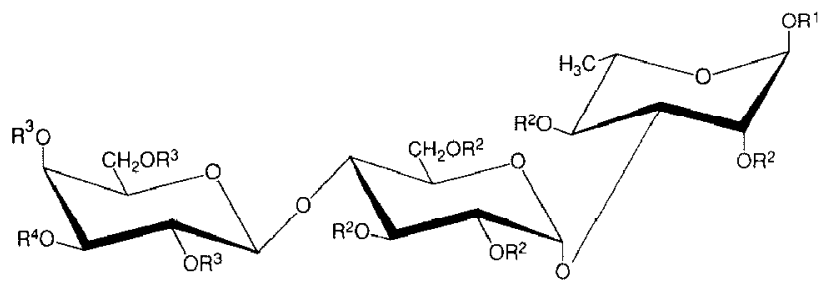

$19 \mathbf{R}^{4}=R^{2}=B n, R^{3}=A C, R^{4}=$ allyl

$20 R^{1}=R^{3} \Rightarrow A C, R^{2}=B n, R^{4}=$ al|yl

$21 R^{1}=M e, R^{2}=B n, R^{3}=A c, R^{4}=$ allyl $\quad C_{2} \mathrm{OBn}$

$22 R^{t}=$ Me. $R^{2}=B n \cdot R^{3}=A C, R^{4}=H$

$1 R^{1}=M e, R^{2}=R^{3}=R^{4}=H$

$25 R^{1}=\mathrm{Me}, \mathrm{A}^{2}=\mathrm{Bn}, \mathrm{R}^{3}=\mathrm{Ac}, \mathrm{A}^{4}=\mathrm{PH}(\mathrm{O}) \mathrm{OCH}_{2} \mathrm{CHOBnCH}_{2} \mathrm{OBn}$

$26 R^{1}=M e, R^{2}=B n, R^{3}=A c_{1} A^{4}=P(O)\left(O^{-+} \mathrm{NHEt}_{3}\right) O C_{2} \mathrm{CHOBnCH}_{2} \mathrm{OBn}$

$2 R^{1}=\mathrm{Me}, R^{2}=\mathrm{R}^{3}=\mathrm{H}, \mathrm{R}^{4}=\mathrm{P}(\mathrm{O})\left(\mathrm{O}^{-} \mathrm{Na}^{+}\right) \mathrm{OCH}_{2} \mathrm{CHOHCH} \mathrm{H}_{2} \mathrm{OH}$

$\mathrm{CH}_{2} \mathrm{OBn}$
$\mathrm{CHOBn}$
$\mathrm{CH}_{2} \mathrm{OR}$
$23 \mathrm{R}=\mathrm{H}$
$24 \mathrm{R}=\mathrm{PH}(\mathrm{O})\left(\mathrm{O}^{-+} \mathrm{NHE}_{5}\right)$

sponding phosphonate using 2-chloro-4H-1,3,2-benzodioxaphosphorin-4-one in acetonitrile-pyridine ${ }^{11,18}$. Condensation of $\mathbf{2 4}$ with 22 gave $74 \%$ of the phosphonic diester 25 in two enantiomeric forms ['H-n.m.r. data: $\delta 6.853\left(J_{\mathrm{H}, \mathrm{P}} 715 \mathrm{~Hz}\right)$ and $6.701\left(J_{\mathrm{H}, \mathrm{P}} 726\right.$ $\mathrm{Hz}), \mathrm{PH}$. Mild oxidation of 25 with iodine in water-pyridine $(\rightarrow \mathbf{2 6}, 92 \%)$, followed by deacetylation, debenzylation, and treatment with Dowex-50 $\left(\mathrm{Na}^{+}\right)$resin, afforded the target methyl glycoside $\mathbf{2}$, which was very labile under mild alkaline conditions. The ${ }^{1} \mathrm{H}$-n.m.r. data for $\mathbf{2}$ are given in Table $\mathrm{I}$.

The syntheses of $\mathbf{3}$ and $\mathbf{4}$ involved the synthons ethyl 2,3,4,6-tetra-O-benzyl-1thio- $\beta$-D-glucopyranoside ${ }^{19}(27)$, methyl 3- $O$-allyl-4,6- $O$-benzylidene- $\beta$-D-galactopyranoside (28), and 2,3,4,6-tetra- $O$-acetyl- $\alpha$-D-glucopyranosyl trichloroacetimidate ${ }^{8}(31)$. Benzylidenation of methyl 3-O-allyl- $\beta$-D-galactopyranoside ${ }^{20}$ using $\alpha, \alpha$-dimethoxytoluene afforded crystalline $28(70 \%)$, which was condensed with 27 in ether, using methyl triflate $^{12}$ as the promoter, to give the disaccharide derivatives $29 \alpha(56 \%)$ and $29 \beta(33 \%)$. Regioselective reductive opening of the 4,6-O-benzylidene ring in $29 \alpha$, as described for 9, yielded $30(76 \%)$, coupling of which with 31 in dichloromethane at $-30^{\circ}$, using trimethylsilyl triflate as the catalyst, gave the trisaccharide derivative $32(90 \%)$. Deallylation $^{21}$ of $32(\rightarrow 33,49 \%)$ followed by further deprotection yielded the target methyl glycoside 3. Coupling of $\mathbf{3 3}$ with $\mathbf{2 4}$ afforded two enantiomers of the phosphonic diester $34(70 \%)$ [ ${ }^{1} \mathrm{H}$-n.m.r. data: $\delta 6.959\left(J_{\text {H.P }} 707 \mathrm{~Hz}\right)$ and $\left.6.901\left(J_{\text {H.P }} 726 \mathrm{~Hz}\right), \mathrm{PH}\right]$. Mild oxidation of 34 with iodine in water-pyridine $(\rightarrow 35,99 \%)$, followed by deacetylation, debenzylation, and treatment with Dowex-50 $\left(\mathrm{Na}^{+}\right)$resin gave the target methyl glycoside 4. The ${ }^{1} \mathrm{H}$-n.m.r. data for $\mathbf{3}$ and $\mathbf{4}$ are given in Table I.

The results of immunological inhibition experiments with $1-4$ will be reported elsewhere.

\section{EXPERIMENTAL}

General methods. - ' $\mathrm{H}-$ N.m.r. spectra (360 and $500 \mathrm{MHz}$ ) were recorded at $25^{\circ}$ with a Bruker HX 360 or AM 500 spectrometer (Bijvoet Center, Utrecht University). 2D double-quantum-filtered ${ }^{1} \mathrm{H}-{ }^{1} \mathrm{H}$ correlation spectra (2D DQF ${ }^{1} \mathrm{H}-{ }^{1} \mathrm{H}$ COSY) were 
TABLE I

500-MHz 'H-N.m.r. data ${ }^{a}$ for the trisaccharide methyl glycosides 1-4

\begin{tabular}{|c|c|c|c|c|c|}
\hline \multirow[t]{2}{*}{ Residue } & \multirow[t]{2}{*}{$\operatorname{Proton}(\mathrm{J})$} & \multicolumn{4}{|c|}{$\delta($ p.p.m. $)(\mathrm{J}$ in $H z)$} \\
\hline & & 1 & 2 & 3 & 4 \\
\hline \multirow[t]{6}{*}{$\alpha-$ Rha } & H-1 $\left(J_{1,2}\right)$ & $4.744(1.8)$ & $4.744(2.1)$ & & \\
\hline & $\mathrm{H}-2\left(J_{2,3}\right)$ & $4.134(2.5)$ & $4.13^{b}$ & & \\
\hline & $\mathrm{H}-3\left(J_{3,4}\right)$ & $3.77(9.8)$ & 3.80 & & \\
\hline & $\mathrm{H}-4\left(J_{4,5}\right)$ & $3.549(9.8)$ & 3.55 & & \\
\hline & H-5 $\left(J_{5,6}\right)$ & 3.71 & $3.72(6.5)$ & & \\
\hline & H-6 & 1.321 & 1.322 & & \\
\hline \multirow[t]{5}{*}{$\alpha$-Glc } & $\mathrm{H}-1\left(J_{1,2}\right)$ & $5.056(3.9)$ & $5.055(4.0)$ & & \\
\hline & $\mathrm{H}-2\left(J_{2,3}\right)$ & $3.630(9.9)$ & 3.63 & & \\
\hline & H-3 $\left(J_{3,4}\right)$ & $3.910(9.5)$ & 3.92 & & \\
\hline & $\mathrm{H}-4\left(J_{4,5}\right)$ & $3.72(10.1)$ & 3.73 & & \\
\hline & 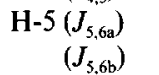 & $\begin{array}{r}4.083(2.4) \\
(3.6)\end{array}$ & 4.09 & & \\
\hline \multirow[t]{5}{*}{$\beta$-Gal } & $\mathrm{H}-1\left(J_{1,2}\right)$ & $4.471(7.8)$ & $4.553(7.9)$ & $4.474(7.9)$ & $4.529(7.9)$ \\
\hline & $\mathrm{H}-2\left(J_{2,3}\right)$ & $3.547(10.0)$ & 3.67 & $3.637(9.8)$ & $3.917(9.9)$ \\
\hline & $\mathrm{H}-3\left(J_{3,4}\right)$ & $3.668(3.4)$ & 4.11 & $3.836(3.3)$ & $4.262^{x}(2.9)$ \\
\hline & $\mathrm{H}-4\left(J_{4,5}\right)$ & $3.929(<1)$ & 4.14 & $4.173(<1)$ & $4.405(<1)$ \\
\hline & $\mathrm{H}-5$ & n.d. & n.d & 3.704 & 3.737 \\
\hline \multirow[t]{6}{*}{$\alpha$-Gic } & H-l $\left(J_{1,2}\right)$ & & & $5.251(3.9)$ & $5.331(3.9)$ \\
\hline & $\mathrm{H}-2\left(J_{2,3}\right)$ & & & $3.523(9.9)$ & $3.503(9.9)$ \\
\hline & $\mathrm{H}-3\left(J_{3,4}\right)$ & & & $3.726(9.3)$ & $3.811(9.3)$ \\
\hline & $\mathrm{H}-4\left(J_{4,5}\right)$ & & & $3.457(10.2)$ & $3.392(10.2)$ \\
\hline & H-5 $\left(J_{5,60}\right)$ & & & $4.025(2.7)$ & $4.101(2.4)$ \\
\hline & $\left(J_{5,6 \mathrm{~b}}\right)$ & & & (3.9) & $(4.5)$ \\
\hline \multirow[t]{7}{*}{$\beta$-Glc } & $\mathrm{H}-1\left(J_{1,2}\right)$ & & & $4.664(8.0)$ & $4.767(7.9)$ \\
\hline & $\mathrm{H}-2\left(J_{2,3}\right)$ & & & $3.370(9.3)$ & $3.319(9.4)$ \\
\hline & H-3 $\left(J_{3,4}\right)$ & & & $3.505(8.7)$ & $3.529(8.6)$ \\
\hline & $\mathrm{H}-4\left(J_{4,5}\right)$ & & & $3.396(9.8)$ & $3.398(9.8)$ \\
\hline & H-5 $\left(J_{5,6 \mathrm{a}}\right)$ & & & $3.440(2.1)$ & $3.445(2.2)$ \\
\hline & H-6a $\left(J_{6 \mathrm{a}, 6 \mathrm{~b}}\right)$ & & & $3.908(-12.3)$ & $3.909(-12.2)$ \\
\hline & $\mathrm{H}-6 \mathrm{~b}\left(J_{2,6 \mathrm{~b}}\right)$ & & & $3.730(5.4)$ & $3.728(5.6)$ \\
\hline $\mathrm{OCH}_{3}$ & & 3.406 & 3.406 & 3.564 & 3.570 \\
\hline \multirow[t]{4}{*}{ Glycerol } & $\begin{array}{r}\text { H-la }\left(J_{1 \mathrm{a}, 2}\right) \\
\left(J_{1 \mathrm{a} .1 \mathrm{~b}}\right)\end{array}$ & & 3.68 & & $\begin{aligned} & 3.689(4.3) \\
&(-11.8)\end{aligned}$ \\
\hline & H-1b $\left(J_{1 \mathrm{~b}, 2}\right)$ & & 3.61 & & $3.607(5.8)$ \\
\hline & $\mathrm{H}-2$ & & 3.90 & & 3.92 \\
\hline & $\mathrm{H}-3 \mathrm{a} / \mathrm{H}-3 \mathrm{~b}$ & & $4.01 / 3.90$ & & $3.996 / 3.92$ \\
\hline
\end{tabular}

${ }^{a}$ Chemical shifts are relative to the signal of internal acetone $\left(\delta 2.225\right.$ p.p.m. in $\left.\mathrm{D}_{2} \mathrm{O}\right) .{ }^{b}$ Chemical shift values with two decimals are deduced directly from $2 \mathrm{D}$ COSY and HOHAHA measurements. ${ }^{c} J_{3, \mathrm{P}} 9.5 \mathrm{~Hz}$. 


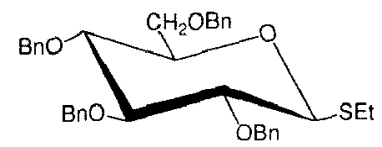

27
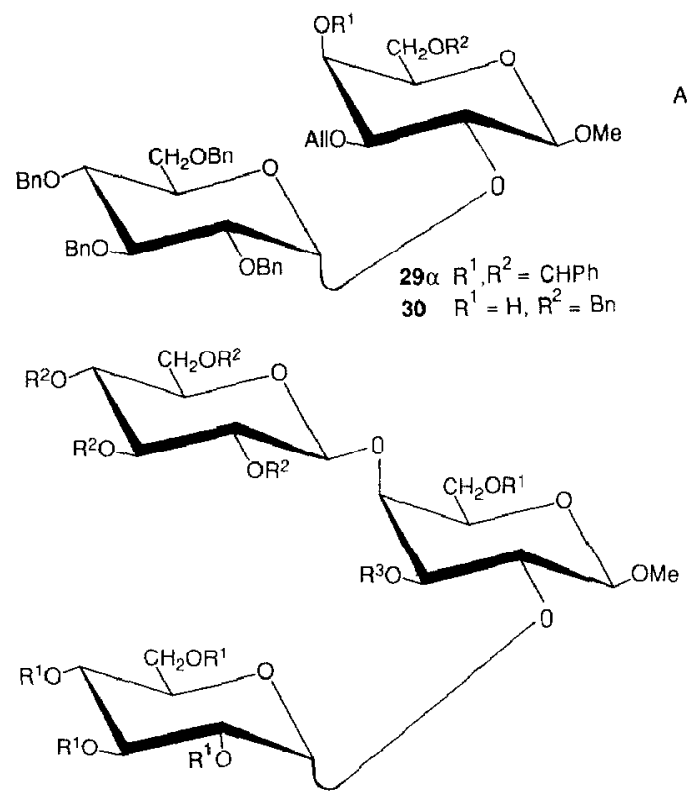

$32 R^{1}=B n, R^{2}=A C, R^{3}=$ allyl

$33 R^{1}=B n, R^{2}=A C, R^{3}=H$

$3 R^{1}=R^{2}=R^{3}=H$

$34 R^{1}=B n_{1} R^{2}=A C, R^{3}=\mathrm{PH}(\mathrm{O}) \mathrm{OCH}_{2} \mathrm{CHOBnCH}_{2} \mathrm{OBn}$

$35 R^{1}=B n, R^{2}=A C, R^{3}=P(O)\left(O^{-1} \mathrm{NHEt}_{3}\right) \mathrm{OCH}_{2} \mathrm{CHOBnCH}_{2} \mathrm{OBn}$

$4 \mathrm{R}^{1}=\mathrm{R}^{2}=\mathrm{H}, \mathrm{R}^{3}=\mathrm{P}(\mathrm{O})\left(\mathrm{O}^{-} \mathrm{Na}^{+}\right) \mathrm{OCH}_{2} \mathrm{CHOHCH} \mathrm{H}_{2} \mathrm{OH}$

recorded in the phase-sensitive mode ${ }^{15}$, and $2 \mathrm{D}$ homonuclear Hartmann-Hahn spectra (2D HOHAHA) with a MLEV-17 mixing sequence of $120 \mathrm{~ms}^{16} \cdot{ }^{13} \mathrm{C}-\mathrm{N} . \mathrm{m} . \mathrm{r}$. spectra (APT, $50 \mathrm{MHz}$ ) were recorded at $25^{\circ}$ with a Bruker WP 200 spectrometer. Chemical shifts $(\delta)$ are given in p.p.m. relative to the signal for internal $\mathrm{Me}_{4} \mathrm{Si}\left(\mathrm{CDCl}_{3}\right)$ or sodium 4,4-dimethyl-4-silapentane-1-sulfonate $\left(\mathrm{D}_{2} \mathrm{O}\right.$; indirectly to internal acetone, $\left.\delta 2.225\right)$ for ${ }^{1} \mathrm{H}$, and to the signal for internal $\mathrm{Me}_{4} \mathrm{Si}\left(\mathrm{CDCl}_{3}\right.$; indirectly to $\left.\mathrm{CDCl}_{3}, \delta 76.9\right)$ or external $\mathrm{Me}_{4} \mathrm{Si}\left(\mathrm{D}_{2} \mathrm{O}\right.$; indirectly to internal acetone, $\left.\delta 31.55\right)$ for ${ }^{13} \mathrm{C}$.

Column chromatography was performed on Kieselgel 60 (Merck, $<230$ mesh) and fractions were monitored by t.l.c. on Kieselgel $60 \mathrm{~F}_{254}$ (Mcrck). Detcction was effected by charring with $\mathrm{H}_{2} \mathrm{SO}_{4}$ after examination under u.v. light. Optical rotations 
were measured at $20^{\circ}$ with a Perkin-Elmer 241 polarimeter, using a $10-\mathrm{cm} 1-\mathrm{mL}$ cell. Melting points were determined with a Mettler FP 51 instrument. In the work-up procedures, washings were carried out three times with appropriate quantities of water or aq. $5 \%$ sodium hydrogencarbonate unless indicated otherwise. Solvents were evaporated under reduced pressure at $40^{\circ}$ (bath). All solvents were distilled from appropriate drying agents.

Ethyl 4,6-O-benzylidene-1-thio- $\beta$-D-glucopyranoside (7). - A mixture of ethyl 1-thio- $\beta$-D-glucopyranoside $5(2.88 \mathrm{~g}, 12.79 \mathrm{mmol})$ and $p$-toluenesulfonic acid $(100 \mathrm{mg})$ in $N, N$-dimethylformamide $(15 \mathrm{~mL})$ and $\alpha, \alpha$-dimethoxytoluene $(30 \mathrm{~mL})$ was heated for $1 \mathrm{~h}$ at $60^{\circ}$ under reduced pressure ${ }^{6}$, then concentrated. The residue was crystallised from aq. saturated sodium hydrogencarbonate $(75 \mathrm{~mL})$ and recrystallised from EtOAc-light petroleum (b.p. $\left.40-60^{\circ}\right)$ to give $7(2.77 \mathrm{~g}, 69 \%)$, m.p. $145^{\circ},[\alpha]_{\mathrm{D}}-65^{\circ}\left(c 1, \mathrm{CHCl}_{3}\right), R_{\mathrm{F}}$ 0.06 (95:5 $\mathrm{CH}_{2} \mathrm{Cl}_{2}-$ EtOAc); lit. ${ }^{5}$ m.p. $144-147^{\circ},[\alpha]_{\mathrm{D}}+47^{\circ}$ (c 1, $\left.\mathrm{CHCl}_{3}\right)$. N.m.r. data $\left(\mathrm{CDCl}_{3}\right):{ }^{13} \mathrm{C}, \delta 136.5$ and $129.2-126.2\left(\mathrm{C}_{6} \mathrm{H}_{5} \mathrm{CH}\right), 101.8(\mathrm{PhCH}), 86.4(\mathrm{C}-1), 80.2,74.4$, 73.1, and $70.4(\mathrm{C}-2,3,4,5), 68.5(\mathrm{C}-6), 24.6\left(\mathrm{CH}_{3} \mathrm{CH}_{2} \mathrm{~S}\right), 15.1\left(\mathrm{CH}_{3} \mathrm{CH}_{2} \mathrm{~S}\right) ;{ }^{1} \mathrm{H}, \delta 7.508-$ 7.365 (m, $5 \mathrm{H}, \mathrm{Ph}$ ), 5.551 (s, $1 \mathrm{H}, \mathrm{PhCH}), 4.478$ (s, 1 H, H-1), 4.361 (dd, $1 \mathrm{H}, \mathrm{H}-6$ ), 2.804-2.743 (m, $\left.2 \mathrm{H}, \mathrm{CH}_{3} \mathrm{CH}_{2} \mathrm{~S}\right), 1.337$ (t, $\left.3 \mathrm{H}, \mathrm{CH}_{3} \mathrm{CH}_{2} \mathrm{~S}\right) ; J_{1,2} 9.7, J_{5,6} 4.9, J_{6 \mathrm{a}, 6 \mathrm{~b}}-10.6$, $J_{\mathrm{CH}_{2} \mathrm{CH}_{3}} 7.4 \mathrm{~Hz}$.

Anal. Calc. for $\mathrm{C}_{15} \mathrm{H}_{20} \mathrm{O}_{5} \mathrm{~S}: \mathrm{C}, 57.67 ; \mathrm{H}, 6.45$. Found: C, 57.53; H, 6.20.

Ethyl 2,3-di-O-benzyl-4,6-O-benzylidene-1-thio- $\beta$-D-glucopyranoside (8). - A solution of $7(1.46 \mathrm{~g}, 4.68 \mathrm{mmol})$ and benzyl bromide $(1.8 \mathrm{~mL}, 14.9 \mathrm{mmol})$ in dry $N, N$-dimethylformamide $(6 \mathrm{~mL})$ was added to a stirred suspension of sodium hydride $(0.45 \mathrm{~g}, 1.88 \mathrm{mmol})$ in $N, N$-dimethylformamide $(5 \mathrm{~mL})$ at $0^{\circ}$. After $1 \mathrm{~h}, \mathrm{t} .1 . \mathrm{c} .(95: 5$ $\mathrm{CH}_{2} \mathrm{Cl}_{2}-\mathrm{EtOAc}$ ) indicated the disappearance of 7 and a product with $R_{\mathrm{F}} 0.75$. Methanol was added to destroy the excess of sodium hydride, the mixture was poured into ice-water $(300 \mathrm{~mL})$ and extracted with ether $(3 \times 75 \mathrm{~mL})$, and the combined extracts were dried $\left(\mathrm{Na}_{2} \mathrm{SO}_{4}\right)$, filtered, and concentrated. The residue was crystallised from EtOH to yield $8(1.86 \mathrm{~g}, 81 \%)$, m.p. $118^{\circ}$ (from EtOH), $[\alpha]_{\mathrm{D}}-43^{\circ}\left(c 1, \mathrm{CHCl}_{3}\right)$. Column chromatography $\left(97: 3 \mathrm{CH}_{2} \mathrm{Cl}_{2}-\mathrm{EtOAc}\right)$ of the material in the mother liquour yielded more $8(0.31 \mathrm{~g}, 13 \%)$. N.m.r. data $\left(\mathrm{CDCl}_{3}\right):{ }^{13} \mathrm{C}, \delta 128.1-125.9\left(\mathrm{C}_{6} \mathrm{H}_{5} \mathrm{CH}_{2} \mathrm{O}\right.$ and $\left.\mathrm{C}_{6} \mathrm{H}_{5} \mathrm{CH}\right), 101.0(\mathrm{PhCH}), 85.7(\mathrm{C}-1), 82.7,81.5,81.2$, and $70.1(\mathrm{C}-2,3,4,5), 75.8$ and 75.1 (2 $\left.\mathrm{PhCH} \mathrm{CH}_{2} \mathrm{O}\right), 68.6(\mathrm{C}-6), 25.0\left(\mathrm{CH}_{3} \mathrm{CH}_{2} \mathrm{~S}\right), 15.0\left(\mathrm{CH}_{3} \mathrm{CH}_{2} \mathrm{~S}\right) ;{ }^{1} \mathrm{H}, \delta 7.491-7.252(\mathrm{~m}, 15 \mathrm{H}$, $3 \mathrm{Ph}), 5.574(\mathrm{~s}, 1 \mathrm{H}, \mathrm{PhCH}), 4.945,4.882,4.808$, and 4.795 (4 d, each $1 \mathrm{H}, 2 \mathrm{PhCH}_{2} \mathrm{O}$ ), 4.560 (d, 1 H, H-1), 4.354 (dd, $1 \mathrm{H}, \mathrm{H}-6$ ), 3.463 (dd, $1 \mathrm{H}, \mathrm{H}-2$ ), 2.810-2.716 (m, 2 H, $\left.\mathrm{CH}_{3} \mathrm{CH}_{2} \mathrm{~S}\right), 1.318\left(\mathrm{t}, 3 \mathrm{H}, \mathrm{CH}_{3} \mathrm{CH}_{2} \mathrm{~S}\right) ; J_{1,2} 9.8, J_{2,3} 8.1, J_{5,6} 5.0, J_{6 \mathrm{a}, 6 \mathrm{~b}}-10.5, J_{\mathrm{CH}_{2} \mathrm{CH}_{3}} 7.4 \mathrm{~Hz}$. Anal. Calc. for $\mathrm{C}_{29} \mathrm{H}_{32} \mathrm{O}_{5} \mathrm{~S}$ : C, 70.71; H, 6.55. Found: C, 70.72; $\mathrm{H}, 6.53$.

Ethyl 2,3,6-tri-O-benzyl-1-thio- $\beta$-D-glucopyranoside (9). A mixture of $\mathbf{8}(1.86 \mathrm{~g}$, $3.77 \mathrm{mmol})$, borane-trimethylamine complex $(1.68 \mathrm{~g}, 23.03 \mathrm{mmol})$, and powdered molecular sieves $(4 \AA, 5.0 \mathrm{~g})$ in tetrahydrofuran ${ }^{7}(50 \mathrm{~mL})$ was stirred for $1 \mathrm{~h}$. Aluminium (III) chloride $(3.12 \mathrm{~g}, 23.40 \mathrm{mmol})$ was added at $0^{\circ}$ and stirring was continued for $3 \mathrm{~h}$, when t.l.c. (95:5 $\left.\mathrm{CH}_{2} \mathrm{Cl}_{2}-\mathrm{EtOAc}\right)$ showed the ring opening to be complete $\left(\rightarrow \mathbf{8}, \boldsymbol{R}_{\mathrm{F}} 0.48\right)$. The mixture was diluted with $\mathrm{CH}_{2} \mathrm{Cl}_{2}(350 \mathrm{~mL})$, filtered through Celite, washed with $\mathrm{M}$ $\mathrm{H}_{2} \mathrm{SO}_{4}(3 \times 50 \mathrm{~mL})$, water, aq. $5 \%$ sodium hydrogencarbonate, and water, dried 
$\left(\mathrm{Na}_{2} \mathrm{SO}_{4}\right)$, filtered, and concentrated. Column chromatography of the residue gave 9 $\left(1.37 \mathrm{~g}, 73 \%\right.$ ), m.p. $66^{\circ}$ (from EtOH), $[\alpha]_{\mathrm{D}}-38^{\circ}\left(\mathrm{c} 1, \mathrm{CHCl}_{3}\right)$. N.m.r. data $\left(\mathrm{CDCl}_{3}\right) \cdot{ }^{13} \mathrm{C}, \delta$ 138.4 and $128.4-127.6\left(\mathrm{C}_{6} \mathrm{H}_{5} \mathrm{CH}_{2} \mathrm{O}\right), 85.8,85.0,81.1,77.8$, and $71.9(\mathrm{C}-1,2,3,4,5), 75.2(2$ C) and $73.5\left(3 \mathrm{PhCH}_{2} \mathrm{O}\right), 70.4(\mathrm{C}-6), 24.8\left(\mathrm{CH}_{3} \mathrm{CH}_{2} \mathrm{~S}\right) ;{ }^{1} \mathrm{H}, \delta 7.404-7.258(\mathrm{~m}, 15 \mathrm{H}, 3 \mathrm{Ph})$, 4.920, 4.788, 4.739, 4.594, and 4.555 (5 d, 2,1,1, l, and $\left.1 \mathrm{H}, 3 \mathrm{PhCH}_{2} \mathrm{O}\right), 4.485(\mathrm{~d}, 1 \mathrm{H}$, $\mathrm{H}-1), 3.743$ (dd, $1 \mathrm{H}, \mathrm{H}-6 \mathrm{a}), 3.717$ (dd, $1 \mathrm{H}, \mathrm{H}-6 \mathrm{~b}), 2.776-2.693\left(\mathrm{~m}, 2 \mathrm{H}, \mathrm{CH}_{3} \mathrm{CH}_{2} \mathrm{~S}\right.$ ), $1.321\left(\mathrm{t}, 3 \mathrm{H}, \mathrm{CH}_{3} \mathrm{CH}_{2} \mathrm{~S}\right) ; J_{1,2} 9.6, J_{5,6 \mathrm{a}} 4.6, J_{5,6 \mathrm{~b}} 5.1, J_{6 \mathrm{a}, 6 \mathrm{~b}}-10.4, J_{\mathrm{CH}_{2} \mathrm{CH}_{3}} 7.4 \mathrm{~Hz}$.

Anal. Calc. for $\mathrm{C}_{29} \mathrm{H}_{34} \mathrm{O}_{5} \mathrm{~S}: \mathrm{C}, 70.42 ; \mathrm{H}, 6.93$. Found: C, 70.47; H, 7.02.

Ethyl 4-O-acetyl-2,3,6-tri-O-benzyl-1-thio- $\beta$-D-glucopyranoside (10). - A solution of $9(12.0 \mathrm{~g}, 24.3 \mathrm{mmol})$ in pyridine $(60 \mathrm{~mL})$ and acetic anhydride $(60 \mathrm{~mL})$ was stirred for $16 \mathrm{~h}$ at room temperature, then concentrated, and toluene, EtOH, and $\mathrm{CII}_{2} \mathrm{Cl}_{2}$ (each $3 \times 50 \mathrm{~mL}$ ) were evaporated from the residue. Column chromatography $\left(97: 3 \mathrm{CH}_{2} \mathrm{Cl}_{2}-\mathrm{EtOAc}\right)$ then gave $10(12.8 \mathrm{~g}, 99 \%)$, m.p. $67^{\circ}$ (from EtOH), $[\alpha]_{\mathrm{p}}-19^{\circ}(\mathrm{c} 1$, $\left.\mathrm{CHCl}_{3}\right), R_{\mathrm{F}} 0.70\left(95: 5 \mathrm{CH}_{2} \mathrm{Cl}_{2}-\mathrm{EtOAc}\right) .{ }^{13} \mathrm{C}-\mathrm{N}$.m.r. data $\left(\mathrm{CDCl}_{3}\right): \delta 138.1-137.6$ and 128.2-127.5 $\left(\mathrm{C}_{6} \mathrm{H}_{5} \mathrm{CH}_{2} \mathrm{O}\right), 84.8,83.6,81.3,77.3$, and $70.9(\mathrm{C}-1,2,3,4,5), 75.4,75.2$, and $73.4\left(3 \mathrm{PhCH}_{2} \mathrm{O}\right), 69.8(\mathrm{C}-6), 24.8\left(\mathrm{CH}_{3} \mathrm{CH}_{2} \mathrm{~S}\right), 20.6\left(\mathrm{COCH}_{3}\right), 15.0\left(\mathrm{CH}_{3} \mathrm{CH}_{2} \mathrm{~S}\right)$.

Benzyl 3-O-(4-O-acetyl-2,3,6-tri-O-benzyl- $\alpha, \beta$-D-glucopyranosyl)-2,4-di-Obenzyl- $\alpha$-L-rhamnopyranoside $(11 \alpha \beta)$. - To a stirred solution of $10(1.80 \mathrm{~g}, 3.36 \mathrm{mmol})$, benzyl 2,4-di- $O$-benzyl- $\alpha$-L-rhamnopyranoside ${ }^{4}(6 ; 1.47 \mathrm{~g}, 3.38 \mathrm{mmol})$, and powdered molecular sieves $(4 \AA, 10 \mathrm{~g})$ in dry ether $(60 \mathrm{~mL})$ was added methyl triflate $(1.9 \mathrm{~mL}, 16.8$ mmol). After $18 \mathrm{~h}$, when t.l.c. $\left(95: 5 \mathrm{CH}_{2} \mathrm{Cl}_{2}-\mathrm{EtOAc}\right)$ indicated two products with $R_{\mathrm{F}} 0.69$ $(11 \beta)$ and $0.64(11 \alpha)$, triethylamine $(8 \mathrm{~mL})$ was added, and stirring was continued for 10 min. The mixture was then filtered through Celite and concentrated. Column chromatography $\left(97: 3 \mathrm{CH}_{2} \mathrm{Cl}_{2}-\mathrm{EtOAc}\right)$ of the residue gave $11 \beta$, isolated as a syrup $(0.94 \mathrm{~g}$, $31 \%),[\alpha]_{\mathrm{D}}-32^{\circ}\left(\mathrm{c} 1, \mathrm{CHCl}_{3}\right)$, and $11 \alpha$, isolated as a $\operatorname{syrup}(1.72 \mathrm{~g}, 57 \%),[\alpha]_{\mathrm{b}}+10^{\circ}(c 1$, $\left.\mathrm{CHCl}_{3}\right)$. N.m.r. data $\left(\mathrm{CDCl}_{3}\right): 11 \beta{ }^{13} \mathrm{C}, \delta 169.5\left(\mathrm{COCH}_{3}\right), 138.4-137.1$ and $128.0-127.3$ $\left(\mathrm{C}_{6} \mathrm{H}_{5} \mathrm{CH}_{2} \mathrm{O}\right), 103.2\left(\mathrm{C}-1^{\prime}\right), 97.5(\mathrm{C}-1), 20.6\left(\mathrm{COCH}_{3}\right), 17.7(\mathrm{C}-6) ;{ }^{1} \mathrm{H}, \delta 7.373-7.186(\mathrm{~m}$, $30 \mathrm{H}, 6 \mathrm{Ph}), 4.962\left(\mathrm{t}, 1 \mathrm{H}, \mathrm{H}-4^{\prime}\right), 4.268(\mathrm{dd}, 1 \mathrm{H}, \mathrm{H}-3), 3.933$ (dd, $\left.1 \mathrm{H}, \mathrm{H}-2\right), 3.763(\mathrm{~m}, 1 \mathrm{H}$, $\mathrm{H}-5), 1.827(\mathrm{~s}, 3 \mathrm{H}, \mathrm{Ac}), 1.330$ (d, $3 \mathrm{H}, \mathrm{H}-6,6,6) ; J_{1,2} 1.8, J_{2.3} 3.2, J_{3.4}=J_{4,5}=9.4, J_{5.6} 6.1$, $J_{3^{\prime} 4^{\prime}}=J_{4^{\prime} 5^{\prime}}=9.4 \mathrm{~Hz} ; 11 \alpha{ }^{13} \mathrm{C}, \delta 169.3\left(\mathrm{COCH}_{3}\right), 138.2-137.7$ and $128.2-127.4$ $\left(\mathrm{C}_{6} \mathrm{H}_{5} \mathrm{CH}_{2} \mathrm{O}\right), 97.0(\mathrm{C}-1), 94.4\left(\mathrm{C}-1^{\prime}\right), 79.8,79.2,79.1,75.8,74.9,70.1,68.5$, and 68.4 $\left(\mathrm{C}-2,3,4,5,2^{\prime}, 3^{\prime}, 4^{\prime}, 5^{\prime}\right), 75.1,74.8,73.3,73.1(2 \mathrm{C}), 68.7$, and $68.1\left(6 \mathrm{PhCH}_{2} \mathrm{O}\right.$ and $\left.\mathrm{C}-6^{\prime}\right)$, $20.6\left(\mathrm{COCH}_{3}\right), 17.9(\mathrm{C}-6) ;{ }^{1} \mathrm{H}, \delta 7.324-7.188(\mathrm{~m}, 30 \mathrm{H}, 6 \mathrm{Ph}), 5.153\left(\mathrm{~d}, 1 \mathrm{H}, \mathrm{H}-1^{\prime}\right), 5.113$ (t, $\left.1 \mathrm{H}, \mathrm{H}-4^{\prime}\right), 4.818(\mathrm{~s}, 1 \mathrm{H}, \mathrm{H}-1), 4.175$ (dd, $\left.1 \mathrm{H}, \mathrm{H}-3\right), 4.080\left(\mathrm{~m}, 1 \mathrm{H}, \mathrm{H}-5^{\prime}\right), 4.019$ (t, $1 \mathrm{H}$, H-3'), 3.759 (m, 1 H, H-5), 3.908 (dd, 1 H, H-2), 3.679 (dd, 1 H, H-2'), 3.678 (t, 1 H, H-4), 3.328 (dd, 1 H, H-6'a), 3.193 (dd, 1 H, H-6'b), 1.716 (s, 3 H, Ac), 1.334 (d, 3 H, H-6,6,6); $J_{1,2}<1, J_{2,3} 3.0, J_{3,4}=J_{4,5}=9.3, J_{5,6} 6.1, J_{1^{\prime}, 2^{\prime}} 3.4, J_{2^{\prime}, 3^{\prime}} 9.6, J_{3^{\prime}, 4^{\prime}}=J_{4^{\prime}, 5^{\prime}}=9.5, J_{5^{\prime}, 6^{\prime} \mathrm{a}} 2.7, J_{5^{\prime}, 6^{\prime} \mathrm{b}}$ $4.1, J_{6 ' a .6 \mathrm{~b}}-11.0 \mathrm{~Hz}$.

Anal. Calc. for $\mathrm{C}_{56} \mathrm{H}_{60} \mathrm{O}_{11}: \mathrm{C}, 73.99 ; \mathrm{H}, 6.65$. Found $11 \propto: \mathrm{C}, 73.65 ; \mathrm{H}, 6.78$. Found $11 \beta: \mathrm{C}, 74.35 ; \mathrm{H}, 6.94$.

Benzyl 2,4-di-O-benzyl-3-O-(2,3,6-tri-O-benzyl- $\alpha-\mathrm{D}-g l$ lucopyranosyl $)-\alpha-\mathrm{L}-$ rhamnopyranoside (12). - To a solution of $11 \propto(6.5 \mathrm{~g}, 7.2 \mathrm{mmol})$ in methanol $(60 \mathrm{~mL})$ was added sodium methoxide to $\mathrm{pH} 10$, and the mixture was stirred overnight. T.1.c. 
(95:5 $\left.\mathrm{CH}_{2} \mathrm{Cl}_{2}-\mathrm{EtOAc}\right)$ then showed the deacetylation to be complete $\left(\rightarrow 12, R_{\mathrm{F}} 0.59\right)$, Dowex-50 $\left(\mathrm{H}^{+}\right)$resin was added, and the mixture was filtered and concentrated. Column chromatography $\left(97: 3 \mathrm{CH}_{2} \mathrm{Cl}_{2}-\mathrm{EtOAc}\right)$ of the residue afforded 12 , isolated as a $\left.\operatorname{syrup}(6.1 \mathrm{~g}, 98 \%),[\alpha]_{\mathrm{D}}-3^{\circ}(c 0.7, \mathrm{CHCl})_{3}\right)$ N.m.r. data $\left(\mathrm{CDCl}_{3}\right):{ }^{13} \mathrm{C}, \delta 138.5-137.2$ and 128.3-127.5 ( $\left.\left.\mathrm{C}_{6} \mathrm{H}_{5} \mathrm{CH}_{2} \mathrm{O}\right), 97.1(\mathrm{C}-1), 95.0\left(\mathrm{C}^{\prime}\right)^{\prime}\right), 81.1,79.9,79.2,76.1,75.3,70.9,70.0$, and $68.2\left(\mathrm{C}-2,3,4,5,2^{\prime}, 3^{\prime}, 4^{\prime}, 5^{\prime}\right), 17.9(\mathrm{C}-6) ;{ }^{1} \mathrm{H}, \delta 7.402-7.127(\mathrm{~m}, 30 \mathrm{H}, 6 \mathrm{Ph}), 5.170(\mathrm{~d}, 1$ H, H-1'), 4.152 (dd, 1 H, H-3), 3.987 (m, 1 H, H-5'), 3.595 (dd, $\left.1 \mathrm{H}, \mathrm{H}-2^{\prime}\right), 3.543(\mathrm{dd}, 1 \mathrm{H}$, H-6'b), 3.494 (dd, $\left.1 \mathrm{H}, \mathrm{H}-6^{\prime} \mathrm{a}\right), 1.322$ (d, $\left.3 \mathrm{H}, \mathrm{H}-6,6,6\right) ; J_{2,3} 2.9, J_{3,4} 8.9, J_{5,6} 6.0, J_{l^{\prime}, 2^{\prime}} 3.4$, $J_{2^{\prime}, 3^{\prime}} 9.6, J_{4^{\prime}, 5^{\prime}} 9.8, J_{5^{\prime}, 6^{\prime} \mathrm{a}} 3.6, J_{5^{\prime}, 6^{\prime} \mathrm{b}} 3.8, J_{6^{\prime}, 6^{\prime} \mathrm{b}}-10.5 \mathrm{~Hz}$.

Anal. Calc. for $\mathrm{C}_{54} \mathrm{H}_{58} \mathrm{O}_{10}: \mathrm{C}, 74.81 ; \mathrm{H}, 6.74$. Found: C, 74.60; $\mathrm{H}, 6.81$.

4-Methoxybenzyl 2,3,4,6-tetra-O-acetyl- $\beta$-D-galactopyranoside (13). - - A solution of 2,3,4,6-tetra- $O$-acetyl- $\alpha$-D-galactopyranosyl trichloroacetimidate ${ }^{8}(5.1 \mathrm{~g}, 10.4$ mmol), 4-methoxybenzyl alcohol $(2.5 \mathrm{~mL}, 20.8 \mathrm{mmol})$, and molecular sieves ( $4 \AA, 10 \mathrm{~g})$ in dry $\mathrm{CH}_{2} \mathrm{Cl}_{2}(40 \mathrm{~mL})$ was stirred for $1 \mathrm{~h}$ under $\mathrm{N}_{2}$. A solution of trimethylsilyl triflate $(38 \mu \mathrm{L}, 0.21 \mathrm{mmol})$ in dry $\mathrm{CH}_{2} \mathrm{Cl}_{2}(1 \mathrm{~mL})$ was added at $-30^{\circ}$. After $10 \mathrm{~min}$, t.l.c. $(85: 15$ $\mathrm{CH}_{2} \mathrm{Cl}_{2}$ EtOAc) indicated the reaction to be complete $\left(\rightarrow 13, R_{\mathrm{F}} 0.25\right)$, pyridine $(2 \mathrm{~mL})$ was added, and the mixture was filtered through Celite and concentrated. Column chromatography $\left(8: 2 \mathrm{CH}_{2} \mathrm{Cl}_{2}-\mathrm{EtOAc}\right)$ of the residue gave 13 , isolated as a syrup $(3.5 \mathrm{~g}$, $73 \%),[\alpha]_{\mathrm{D}}-24^{\circ}\left(\mathrm{c} 1, \mathrm{CHCl}_{3}\right)$. N.m.r. data $\left(\mathrm{CDCl}_{3}\right):{ }^{13} \mathrm{C}, \delta 169.7(2 \mathrm{C}), 169.4$, and $168.7(4$ $\left.\mathrm{COCH}_{3}\right), 158.9,128.9(2 \mathrm{C}), 128.2$, and $113.3(2 \mathrm{C})\left(\mathrm{MeOC}_{6} \mathrm{H}_{4} \mathrm{CH}_{2} \mathrm{O}\right), 98.8(\mathrm{C}-1), 70.4$, 70.1, 68.3, and $66.6(\mathrm{C}-2,3,4,5), 69.8\left(\mathrm{MeOC}_{6} \mathrm{H}_{4} \mathrm{CH}_{2} \mathrm{O}\right), 60.8(\mathrm{C}-6), 54.6\left(\mathrm{CH}_{3} \mathrm{OC}_{6} \mathrm{H}_{4}-\right.$ $\left.\mathrm{CH}_{2} \mathrm{O}\right), 20.1-19.9\left(\mathrm{COCH}_{3}\right) ;{ }^{1} \mathrm{H}, \delta 7.218$ and $6.880\left(2 \mathrm{~d}\right.$, each $\left.2 \mathrm{H}, \mathrm{MeOC}_{6} \mathrm{H}_{4} \mathrm{CH}_{2} \mathrm{O}\right)$, 5.382 (bd, $1 \mathrm{H}, \mathrm{H}-4), 5.256$ (dd, $1 \mathrm{H}, \mathrm{H}-2), 4.976$ (dd, $1 \mathrm{H}, \mathrm{H}-3), 4.835$ and 4.575 (2 d, each $\left.1 \mathrm{H}, \mathrm{MeOC}_{6} \mathrm{H}_{4} \mathrm{CH}_{2} \mathrm{O}\right), 4.484(\mathrm{~d}, 1 \mathrm{H}, \mathrm{H}-1), 4.219$ (dd, $\left.1 \mathrm{H}, \mathrm{H}-6 \mathrm{a}\right), 4.152$ (dd, $1 \mathrm{H}$, $\mathrm{H}-6 \mathrm{~b}), 3.875$ (m, $1 \mathrm{H}, \mathrm{H}-5), 3.816$ (s, $\left.3 \mathrm{H}, \mathrm{CH}_{3} \mathrm{OC}_{6} \mathrm{H}_{4} \mathrm{CH}_{2} \mathrm{O}\right), 2.157,2.072,2.004$, and $1.974(4 \mathrm{~s}$, each $1 \mathrm{H}, 4 \mathrm{Ac}) ; J_{1,2} 7.9, J_{2,3} 10.4, J_{3,4} 3.5, J_{4,5}<1, J_{5,6 \mathrm{a}} 6.5, J_{5,6 \mathrm{~b}} 6.9, J_{6 \mathrm{a}, 6 \mathrm{~b}}-11.2$ Hz.

Anal. Calc. for $\mathrm{C}_{22} \mathrm{H}_{28} \mathrm{O}_{11}: \mathrm{C}, 56.41 ; \mathrm{H}, 6.02$. Found: $\mathrm{C}, 56.85 ; \mathrm{H}, 6.07$.

4-Methoxybenzyl 2,4,6-tri-O-acetyl-3-O-allyl- $\beta$-D-galactopyranoside (16). - To a solution of $13(14.7 \mathrm{~g}, 32.4 \mathrm{mmol})$ in $\mathrm{MeOH}(125 \mathrm{~mL})$ was added sodium methoxide to $\mathrm{pH} 10$. After $16 \mathrm{~h}$, Dowex $-50\left(\mathrm{H}^{+}\right)$resin was added, and the solution was filtered and concentrated. A solution of the product $14\left(R_{\mathrm{F}} 0.25,9: 1 \mathrm{CH}_{2} \mathrm{Cl}_{2}-\mathrm{MeOH}\right)$ and dibutyltin oxide $(8.1 \mathrm{~g}, 32.4 \mathrm{mmol})$ in benzene $(150 \mathrm{~mL})$ was boiled under reflux for $16 \mathrm{~h}$ in a Soxhlet apparatus containing molecular sieves $(3 \AA)$. Then tetrabutylammonium iodide $(11.8 \mathrm{~g}, 32.4 \mathrm{mmol})$ and allyl bromide $(4.8 \mathrm{~mL}, 55.8 \mathrm{mmol})$ were added, and boiling was continued for $2 \mathrm{~h}$, when t.l.c. $\left(7: 3 \mathrm{CH}_{2} \mathrm{Cl}_{2}\right.$-acetone) showed the formation of $15\left(R_{\mathrm{F}}\right.$ 0.61 ). The mixture was concentrated and column chromatography of the residue afforded 15. A solution of 15 in pyridine $(75 \mathrm{~mL})$ and acetic anhydride $(40 \mathrm{~mL})$ was stirred overnight to yield 16 as indicated by t.l.c. $\left(8: 2 \mathrm{CH}_{2} \mathrm{Cl}_{2}-\mathrm{EtOAc}, R_{\mathrm{F}} 0.70\right)$. The mixture was concentrated, and toluene, $\mathrm{EtOH}$, and $\mathrm{CH}_{2} \mathrm{Cl}_{2}$ (each $3 \times 50 \mathrm{~mL}$ ) were evaporated from the residue. Crystallisation from EtOH afforded $16(5.2 \mathrm{~g}, 35 \%)$, m.p. $87^{\circ}$ (from EtOH), $[\alpha]_{\mathrm{D}}-26^{\circ}\left(c 0.6, \mathrm{CHCl}_{3}\right)$. Column chromatography $\left(85: 15 \mathrm{CH}_{2} \mathrm{Cl}_{2}-\right.$ acetone) of the material in the mother liquour afforded more $16(1.1 \mathrm{~g}, 7 \%)$. N.m.r. data 
$\left(\mathrm{CDCl}_{3}\right):{ }^{13} \mathrm{C}, \delta 170.2,170.1$, and $169.2\left(3 \mathrm{COCH}_{3}\right), 159.2,129.2(2 \mathrm{C}), 128.7$, and $113.6(2$ C) $\left(\mathrm{MeOC}_{6} \mathrm{H}_{4} \mathrm{CH}_{2} \mathrm{O}\right), 133.9\left(\mathrm{H}_{2} \mathrm{C}=\mathrm{CHCH}_{2} \mathrm{O}\right), 117.0\left(\mathrm{H}_{2} \mathrm{C}=\mathrm{CHCH}_{2} \mathrm{O}\right), 99.1$ (C-1), $76.4,70.7,70.2$, and $65.9(\mathrm{C}-2,3,4,5), 70.3$ and $69.8\left(\mathrm{H}_{2} \mathrm{C}-\mathrm{CHCH}_{2} \mathrm{O}\right.$ and $\left.\mathrm{MeOC}_{6} \mathrm{H}_{4} \mathrm{CH}_{2} \mathrm{O}\right), 61.8(\mathrm{C}-6), 55.0\left(\mathrm{CH}_{3} \mathrm{OC}_{6} \mathrm{H}_{4} \mathrm{CH}_{2} \mathrm{O}\right), 20.6-20.5\left(\mathrm{COCH}_{3}\right) ;{ }^{1} \mathrm{H}, \delta 7.220$ and $6.872\left(2 \mathrm{~d}\right.$, each $\left.2 \mathrm{H}, \mathrm{McOC}_{6} \mathrm{H}_{4} \mathrm{CH}_{2} \mathrm{O}\right), 5.763\left(\mathrm{~m}, 1 \mathrm{H}, \mathrm{H}_{2} \mathrm{C}=\mathrm{CHCH}_{2} \mathrm{O}\right), 5.405$ (bd, 1 $\mathrm{H}, \mathrm{H}-4), 4.819$ and $4.568\left(2 \mathrm{~d}\right.$, each $\left.1 \mathrm{H}, \mathrm{MeOC}_{6} \mathrm{H}_{4} \mathrm{CH}_{2} \mathrm{O}\right), 4.410(\mathrm{~d}, 1 \mathrm{H}, \mathrm{H}-1), 4.103$ and $3.885\left(2 \mathrm{~m}\right.$, each $\left.1 \mathrm{H}, \mathrm{H}_{2} \mathrm{C}=\mathrm{CHCH}_{2} \mathrm{O}\right), 3.807\left(\mathrm{~s}, 3 \mathrm{H}, \mathrm{CH}_{3} \mathrm{OC}_{6} \mathrm{H}_{4} \mathrm{CH}_{2} \mathrm{O}\right), 3.774(\mathrm{~m}, 1 \mathrm{H}$, H-5), 3.471 (dd, $1 \mathrm{H}, \mathrm{H}-3), 2.142,2.090$, and 2.036 ( $3 \mathrm{~s}$, each $3 \mathrm{H}, 3 \mathrm{Ac}$ ); $J_{1.2} 8.1, J_{2,3} 10.0$, $J_{3,4} 3.5, J_{4,5}<1, J_{5,6 \mathrm{a}} \approx J_{5,6 \mathrm{~b}} \approx 6.5 \mathrm{~Hz}$.

Anal. Calc. for $\mathrm{C}_{23} \mathrm{H}_{30} \mathrm{O}_{10}: \mathrm{C}, 59.22 ; \mathrm{H}, 6.48$. Found: C, 58.81; H, 6.33.

2,4,6-Tri-O-acetyl-3-O-allyl- $\alpha, \beta$-D-galactopyranose (17). - To a solution of 16 $(2.0 \mathrm{~g}, 4.4 \mathrm{mmol})$ in acetonitrile $(45 \mathrm{~mL})$ and water $(5 \mathrm{~mL})$ was added ceric ammonium nitrate $(4.9 \mathrm{~g}, 8.8 \mathrm{mmol})$. When t.l.c. $\left(8: 2 \mathrm{CH}_{2} \mathrm{Cl}_{2}-\mathrm{EtOAc}\right)$ indicated the reaction to be complete ( $3 \mathrm{~h} ; 17, R_{\mathrm{F}} 0.26$ ), the mixture was diluted with $\mathrm{CH}_{2} \mathrm{Cl}_{2}(300 \mathrm{~mL}$ ), washed with water, aq. concentrated sodium hydrogensulfite $(2 \times 50 \mathrm{~mL})$, aq. $5 \%$ sodium hydrogencarbonate $(2 \times 50 \mathrm{~mL})$, and water, dried $\left(\mathrm{Na}_{2} \mathrm{SO}_{4}\right)$, filtered, and concentrated. Column chromatography of the residue afforded 17 , isolated as a syrup $(1.2 \mathrm{~g}, 81 \%)$, having analytical data as reported previously".

Benzyl 2,4-di-O-benzyl-3-O-/2,3,6-tri-O-benzyl-4-O-(2,4,6-tri-O-acetyl-3-Oallyl- $\beta$-D-galactopyranosyl)- $\alpha$-D-glucopyranosyl $]-\alpha-\mathrm{L}-$ rhamnopyranoside $(19)$. - To a solution of 18 (ref. 11$)\left(0.55 \mathrm{~g}, 0.63 \mathrm{mmol}\right.$ ) and $12(0.39 \mathrm{~g}, 0.80 \mathrm{mmol})$ in dry $\mathrm{CH}_{2} \mathrm{Cl}_{2}$ containing molecular sieves ( $4 \AA, 3 \mathrm{~g}$ ) was added a solution of trimethylsilyl triflate (43 $\mu \mathrm{L}, 0.24 \mathrm{mmol})$ in $\mathrm{CH}_{2} \mathrm{Cl}_{2}(2 \mathrm{~mL})$ at $-30^{\circ}$. When t.l.c. $\left(29: 2 \mathrm{CH}_{2} \mathrm{Cl}_{2}-\mathrm{EtOAc}\right)$ indicated the disappearance of $18\left(R_{\mathrm{F}} 0.78\right)$, pyridine $(2 \mathrm{~mL})$ was added, and the mixture was filtered through Celite and concentrated. Column chromatography of the residue gave 19 , isolated as a syrup $(0.57 \mathrm{~g}, 73 \%),[\alpha]_{\mathrm{D}}-1^{\circ}\left(c 1, \mathrm{CHCl}_{3}\right), R_{\mathrm{F}} 0.59$. N.m.r. data $\left(\mathrm{CDCl}_{3}\right)$ : ${ }^{13} \mathrm{C}, \delta 169.8(2 \mathrm{C})$ and $168.6\left(3 \mathrm{COCH}_{3}\right), 138.9-137.2$ and $128.1-126.8\left(\mathrm{C}_{6} \mathrm{H}_{5} \mathrm{CH}_{2} \mathrm{O}\right)$, $133.8\left(\mathrm{H}_{2} \mathrm{C}=\mathrm{CHCH}_{2} \mathrm{O}\right), 116.5\left(\mathrm{H}_{2} \mathrm{C}=\mathrm{CHCH}_{2} \mathrm{O}\right), 99.9\left(\mathrm{C}-1^{\prime \prime}\right), 97.0(\mathrm{C}-1), 96.2\left(\mathrm{C}-1^{\prime}\right)$, 20.5 and $20.3(2 \mathrm{C})\left(3 \mathrm{COCH}_{3}\right), 17.6(\mathrm{C}-6){ }^{1}{ }^{\mathrm{H}} \mathrm{H}, \delta 7.311-7.055(\mathrm{~m}, 30 \mathrm{H}, 6 \mathrm{Ph}), 5.680(\mathrm{~m}, 1$ $\mathrm{H}, \mathrm{H}_{2} \mathrm{C}=\mathrm{CHCH}_{2} \mathrm{O}$ ), 5.200 (bd, $\left.1 \mathrm{H}, \mathrm{H}-4^{\prime \prime}\right), 5.070\left(\mathrm{~d}, 1 \mathrm{H}, \mathrm{H}-\mathrm{I}^{\prime}\right), 4.925\left(\mathrm{dd}, 1 \mathrm{H}, \mathrm{H}-2^{\prime \prime}\right)$, 4.394 (d, $\left.1 \mathrm{H}, \mathrm{H}-\mathrm{l}^{\prime \prime}\right), 3.107$ (dd, $\left.1 \mathrm{H}, \mathrm{H}-3^{\prime \prime}\right), 1.996,1.937$, and 1.810 (3 s, each $3 \mathrm{H}, 3 \mathrm{Ac}$ ), 1.174 (d, $3 \mathrm{H}, \mathrm{H}-6,6,6), J_{1^{\prime}, 2^{\prime}} 3.6, J_{1^{\prime \prime}, 2^{\prime \prime}} 8.1, J_{2^{\prime \prime}, 3^{\prime \prime}} 10.0, J_{3^{\prime \prime}, 4^{\prime \prime}} 3.4, J_{4^{\prime \prime}, 5^{\prime \prime}}<1 \mathrm{~Hz}$.

Anal. Calc. for $\mathrm{C}_{69} \mathrm{H}_{78} \mathrm{O}_{18}: \mathrm{C}, 69.33 ; \mathrm{H}, 6.58$. Found: $\mathrm{C}, 69.19 ; \mathrm{H}, 6.30$.

l-O-Acetyl-2,4-di-O-benzyl-3-O-[2,3,6-tri-O-benzyl-4-O-(2,4,6-tri-O-acetyl-3O-allyl- $\beta$-D-galactopyranosyl)- $\alpha$-D-glucopyranosyl $-\alpha-\mathrm{L}$-rhamnopyranose (20). - A solution of $\mathrm{H}_{2} \mathrm{SO}_{4}(10 \mu \mathrm{L})$ in acetic anhydride $(0.99 \mathrm{~mL})$ was added to a solution of $19(0.57$ $\mathrm{g}, 0.54 \mathrm{mmol})$ in acetic anhydride $(9 \mathrm{~mL})$ and acetic acid $(5 \mathrm{~mL})$ at $0^{\circ}$. The mixture was stirred for $2 \mathrm{~h}$ at room temperature, poured into ice-water containing aq. concentrated sodium hydrogencarbonate $(300 \mathrm{~mL})$, and, after $16 \mathrm{~h}$, extracted with $\mathrm{CH}_{2} \mathrm{Cl}_{2}(3 \times 50$ $\mathrm{mL})$. The combined extracts were washed with water, dried $\left(\mathrm{Na}_{2} \mathrm{SO}_{4}\right)$, filtered, and concentrated. Column chromatography $\left(9: 1 \mathrm{CH}_{2} \mathrm{Cl}_{2}-\mathrm{EtOAc}\right)$ of the residue gave $\mathbf{2 0}$, isolated as a syrup $(0.48 \mathrm{~g}, 78 \%),[\alpha]_{\mathrm{D}}+12^{\circ}\left(\mathrm{c} \mathrm{l}, \mathrm{CHCl}_{3}\right), R_{\mathrm{r}} 0.35$. N.m.r. data $\left(\mathrm{CDCl}_{3}\right)$ : ${ }^{13} \mathrm{C}, \delta 170.1-168.9\left(\mathrm{COCH}_{3}\right), 139.1-137.8$ and $128.4-127.1\left(\mathrm{C}_{6} \mathrm{H}_{5} \mathrm{CH}_{2} \mathrm{O}\right), 134.0$ 
$\left(\mathrm{H}_{2} \mathrm{C}=\mathrm{CHCH}_{2} \mathrm{O}\right), 116.9\left(\mathrm{H}_{2} \mathrm{C}=\mathrm{CHCH}_{2} \mathrm{O}\right), 100.2\left(\mathrm{C}^{\prime \prime}{ }^{\prime \prime}\right), 96.2\left(\mathrm{C}-1^{\prime}\right), 91.6(\mathrm{C}-1), 20.8-$ $20.6\left(\mathrm{COCH}_{3}\right), 17.9(\mathrm{C}-6) ;{ }^{1} \mathrm{H}, \delta 7.370-7.193(\mathrm{~m}, 25 \mathrm{H}, 5 \mathrm{Ph}), 6.070(\mathrm{~d}, 1 \mathrm{H}, \mathrm{H}-1), 5.764$ $\left(\mathrm{m}, 1 \mathrm{H}, \mathrm{H}_{2} \mathrm{C}=\mathrm{CHCH}_{2} \mathrm{O}\right), 5.279$ and $5.145\left(2 \mathrm{~d}\right.$, each $\left.1 \mathrm{H}, \mathrm{H}_{-1} 1^{\prime}, 4^{\prime \prime}\right), 5.015$ (dd, $1 \mathrm{H}$, $\mathrm{H}-2^{\prime \prime}$ ), 4.496 (d, $\left.1 \mathrm{H}, \mathrm{H}-1^{\prime \prime}\right), 3.208$ (dd, $\left.1 \mathrm{H}, \mathrm{H}-3^{\prime \prime}\right), 2.068,2.003,1.938$, and 1.900 (4 s, each $3 \mathrm{H}, 4 \mathrm{Ac}) ; J_{1,2} 2.2, J_{1^{\prime}, 2^{\prime}} \approx 3, J_{1^{\prime \prime}, 2^{\prime \prime}} 8.1, J_{2^{\prime \prime}, 3^{\prime \prime}} 9.9, J_{3^{\prime \prime}, 4^{\prime \prime}} 3.4, J_{4^{\prime \prime} .5^{\prime \prime}}<1 \mathrm{~Hz}$.

Anal. Calc. for $\mathrm{C}_{64} \mathrm{H}_{74} \mathrm{O}_{14}: \mathrm{C}, 67.00 ; \mathrm{H}, 6.50$. Found: C, 67.04; H, 6.72.

Methyl 2,4-di-O-benzyl-3-O-[2,3,6-tri-O-benzyl-4-O-(2,4,6-tri-O-acetyl-3-O-allyl- $\beta$-D-galactopyranosyl)- $\alpha$-D-glucopyranosyl]- $\alpha$-L-rhamnopyranoside (21). - To a solution of $20(105 \mathrm{mg}, 92 \mu \mathrm{mol})$ in dry $\mathrm{CH}_{2} \mathrm{Cl}_{2}(6 \mathrm{~mL})$, containing $\mathrm{MeOH}(40 \mu \mathrm{L}, 0.98$ $\mathrm{mmol}$ ) and powdered molecular sieves $(3 \AA, 150 \mathrm{mg})$, was added trimethyl triflate ${ }^{13}$ (53 $\mu \mathrm{L}, 0.29 \mathrm{mmol}$ ). After $15 \mathrm{~min}$, t.l.c. [7:3 light petroleum (b.p. $40-60^{\circ}$ )-EtOAc] indicated the conversion of $20\left(R_{\mathrm{F}} 0.20\right)$ into $21\left(R_{\mathrm{F}} 0.36\right)$, pyridine was added $(5 \mathrm{~mL})$, and the mixture was diluted with $\mathrm{CH}_{2} \mathrm{Cl}_{2}(50 \mathrm{~mL})$, filtered through Celite, and concentrated. Column chromatography of the residue afforded 21 , isolated as a syrup (64 $\mathrm{mg}, 62 \%$ ), $[\alpha]_{\mathrm{D}}+21^{\circ}\left(c 1, \mathrm{CHCl}_{3}\right)$. N.m.r. data $\left(\mathrm{CDCl}_{3}\right):{ }^{13} \mathrm{C}, \delta 170.2-168.9\left(\mathrm{COCH}_{3}\right), 139.1-137.9$ and 128.3-127.0 $\left(\mathrm{C}_{6} \mathrm{H}_{5} \mathrm{CH}_{2} \mathrm{O}\right), 134.0\left(\mathrm{H}_{2} \mathrm{C}=\mathrm{CHCH}_{2} \mathrm{O}\right), 116.8\left(\mathrm{H}_{2} \mathrm{C}=\mathrm{CHCH}_{2} \mathrm{O}\right), 100.2$ $\left(\mathrm{C}-1^{\prime \prime}\right), 99.1(\mathrm{C}-1), 96.1\left(\mathrm{C}-1^{\prime}\right), 54.5\left(\mathrm{OCH}_{3}\right), 20.7-20.6\left(\mathrm{COCH}_{3}\right), 17.8(\mathrm{C}-6), J_{\mathrm{C}-1, \mathrm{H}-1} 167$, $\dot{J}_{\mathrm{C}-1^{\prime}, \mathrm{H}-1^{\prime}} 167, J_{\mathrm{C}-1^{\prime \prime}, \mathrm{H}-1^{\prime \prime}} 160 \mathrm{~Hz} ;{ }^{1} \mathrm{H}, \delta 7.378-7.208(\mathrm{~m}, 25 \mathrm{H}, 5 \mathrm{Ph}), 5.759(\mathrm{~m}, 1 \mathrm{H}$, $\left.\mathrm{H}_{2} \mathrm{C}=\mathrm{CHCH}_{2} \mathrm{O}\right), 5.272\left(\mathrm{bd}, 1 \mathrm{H}, \mathrm{H}-4^{\prime \prime}\right), 5.219$ and $5.155(2 \mathrm{~m}$, each $1 \mathrm{H}$, $\mathrm{H}_{2} \mathrm{C}=\mathrm{CHCH}_{2} \mathrm{O}$ ), $5.157\left(\mathrm{~d}, 1 \mathrm{H}, \mathrm{H}-1^{\prime}\right), 4.998\left(\mathrm{dd}, 1 \mathrm{H}, \mathrm{H}-2^{\prime \prime}\right), 4.485$ (d, $\left.1 \mathrm{H}, \mathrm{H}-\mathrm{l}^{\prime \prime}\right), 3.271$ (s, $3 \mathrm{H}, \mathrm{OMe}$ ), 3.190 (dd, $1 \mathrm{H}, \mathrm{H}-3^{\prime \prime}$ ), 2.063, 2.001, and 1.880 (3 s, each $3 \mathrm{H}, 3 \mathrm{Ac}$ ), 1.237 (d, $3 \mathrm{H}, \mathrm{H}-6,6,6) ; J_{5,6} 5.6, J_{1^{\prime}, 2^{\prime}} 3.7, J_{1^{\prime \prime}, 2^{\prime \prime}} 8.1, J_{2^{\prime \prime}, 3^{\prime \prime}} 9.9, J_{3^{\prime \prime}, 4^{\prime \prime}} 3.4, J_{4^{\prime \prime}, 5^{\prime \prime}}<1 \mathrm{~Hz}$.

Anal. Calc. for $\mathrm{C}_{63} \mathrm{H}_{74} \mathrm{O}_{18}: \mathrm{C}, 67.61 ; \mathrm{H}, 6.66$. Found: $\mathrm{C}, 67.64 ; \mathrm{H}, 6.85$.

Methyl 2,4-di-O-benzyl-3-O-[2,3,6-tri-O-benzyl-4-O-(2,4,6-tri-O-acetyl- $\beta$-D-galactopyranosyl)- $\alpha$-D-glucopyranosyl]- $\alpha$-L-rhamnopyranoside (22). - A mixture of 21 (61 mg, $54 \mu \mathrm{mol}$ ), palladium(II) chloride ${ }^{14}$ (54 mg, $0.30 \mathrm{mmol}$ ), and sodium acetate (47 $\mathrm{mg}, 0.57 \mathrm{mmol})$ in acetic acid $(1.4 \mathrm{~mL})$ was sonicated for $22 \mathrm{~h}$. The mixture was diluted with $\mathrm{CH}_{2} \mathrm{Cl}_{2}(50 \mathrm{~mL})$, filtered through Celite, washed with water, aq. $5 \%$ sodium hydrogencarbonate, and aq. $5 \%$ sodium chloride, dried $\left(\mathrm{Na}_{2} \mathrm{SO}_{4}\right)$, filtered, and concentrated. Column chromatography $\left(9: 1 \mathrm{CH}_{2} \mathrm{Cl}_{2}\right.$-acetone) of the residue gave 22 , isolated as a syrup (44 mg, 75\%), $[\alpha]_{\mathrm{b}}+5^{\circ}\left(c 1, \mathrm{CHCl}_{3}\right), R_{\mathrm{F}}$ 0.42. N.m.r. data $\left(\mathrm{CDCl}_{3}\right)$ : ${ }^{13} \mathrm{C}, \delta$ 171.2, 170.6, and 170.2 (3 $\left.\mathrm{COCH}_{3}\right), 139.1-137.8$ and 128.3-127.1 $\left(\mathrm{C}_{6} \mathrm{H}_{5} \mathrm{CH}_{2} \mathrm{O}\right), 99.8(\mathrm{C}-$ $\left.1^{\prime \prime}\right), 99.0(\mathrm{C}-1), 95.7\left(\mathrm{C}-1^{\prime}\right), 54.6\left(\mathrm{OCH}_{3}\right), 20.8-20.6\left(\mathrm{COCH}_{3}\right), 17.9(\mathrm{C}-6) ;{ }^{1} \mathrm{H}, \delta 7.358$ 7.217 (m, 25 H, $5 \mathrm{Ph}$ ), 5.184 (bd, $\left.1 \mathrm{H}, \mathrm{H}-4^{\prime \prime}\right), 5.162$ (d, $\left.1 \mathrm{H}, \mathrm{H}-1^{\prime}\right), 4.473$ (d, $1 \mathrm{H}, \mathrm{H}-\mathrm{l}^{\prime \prime}$ ), 3.278 (s, 3 H, OMe), 2.095, 1.999, and 1.888 ( $3 \mathrm{~s}$, each $3 \mathrm{H}, 3 \mathrm{Ac}), 1.266$ (d, $3 \mathrm{H}, \mathrm{H}-6,6,6)$; $J_{5,6} 5.8, J_{1^{\prime}, 2^{\prime}} 3.6, J_{1^{\prime \prime}, 2^{\prime \prime}} 7.9, J_{3^{\prime \prime}, 4^{\prime \prime}} 3.6, J_{4^{\prime \prime}, 5^{\prime \prime}}<1 \mathrm{~Hz}$.

Methyl 3-O-(4-O- $\beta$-D-galactopyranosyl- $\alpha$-D-glucopyranosyl)- $\alpha$-L-rhamnopyranoside (1). - To a solution of $22(21 \mathrm{mg}, 20 \mu \mathrm{mol})$ in $\mathrm{MeOH}(5 \mathrm{~mL})$ was added sodium methoxide to $\mathrm{pH} 10$. After $24 \mathrm{~h}$, the mixture was neutralised with Dowex- $50\left(\mathrm{H}^{+}\right)$resin, filtered, and concentrated. A solution of the residue in $\mathrm{EtOH}(5 \mathrm{~mL})$ containing $10 \%$ $\mathrm{Pd} / \mathrm{C}$ (20 mg) was hydrogenolysed for $20 \mathrm{~h}$ at $4 \mathrm{~kg} / \mathrm{cm}^{2}$, filtered through Celite, and concentrated to give 1 , isolated as a white powder $(8 \mathrm{mg}, 79 \%),[\alpha]_{\mathrm{D}}+80^{\circ}\left(c 0.7, \mathrm{H}_{2} \mathrm{O}\right)$. ${ }^{13}$ C-N.m.r. data $\left(\mathrm{D}_{2} \mathrm{O}\right): \delta 104.2$ and $101.8\left(\mathrm{C}-1^{\prime}, 1^{\prime \prime}\right), 96.8(\mathrm{C}-1), 79.4,77.2,76.7,73.9$, 
$72.9,72.4,72.3,71.7,71.5,69.9(2 \mathrm{C})$, and $68.0\left(\mathrm{C}-2,3,4,5,2^{\prime}, 3^{\prime}, 4^{\prime}, 5^{\prime}, 2^{\prime \prime}, 3^{\prime \prime}, 4^{\prime \prime}, 5^{\prime \prime}\right), 62.4$ and $60.9\left(\mathrm{C}^{-} 6^{\prime}, 6^{\prime \prime}\right), 56.1\left(\mathrm{OCH}_{3}\right), 18.0(\mathrm{C}-6)$. For the ${ }^{1} \mathrm{H}$-n.m.r. data, see Table I.

1,2-Di-O-henzyl-sn-glycerol 3-(triethylammonium phosphonate) (24). - To a solution of 1,2-di- $O$-benzyl-sn-glycerol ${ }^{17}(157 \mathrm{mg}, 0.58 \mathrm{mmol})$ in $4: 1$ acetonitrilepyridine $(3 \mathrm{~mL})$ was added a solution of 2-chloro-4H-1,3,2-benzodioxaphosphorin-4one (145 mg, $0.72 \mathrm{mmol})$ in acetonitrile $(0.6 \mathrm{~mL})$. After $1 \mathrm{~h}$, t.1.c. $\left(9: 1 \mathrm{CH}_{2} \mathrm{Cl}_{2}\right.$-acetone $)$ indicated a partial conversion of the starting compound into $24\left(R_{\mathrm{F}} 0\right)$, which could not be improved. Water $(1 \mathrm{~mL})$ was added, and the mixture was diluted with $\mathrm{CH}_{2} \mathrm{Cl}_{2}(100$ $\mathrm{mL})$, washed with $\mathrm{M}$ triethylammonium hydrogencarbonate $(2 \times 15 \mathrm{~mL})$, dried $\left(\mathrm{Na}_{2} \mathrm{SO}_{4}\right)$, filtered, and concentrated. Column chromatography (9:1:0.1 $\mathrm{CH}_{2} \mathrm{Cl}_{2}$-acetone-triethylamine, followed by $9: 1: 0.1 \mathrm{CH}_{2} \mathrm{Cl}_{2}-\mathrm{MeOH}$-triethylamine) of the residue yielded 24 , isolated as a syrup $(58 \mathrm{mg}, 23 \%)$. N.m.r. data $\left(\mathrm{CDCl}_{3}\right):{ }^{13} \mathrm{C}, \delta$ 138.2, 137.8, and 127.7-127.1 $\left(\mathrm{C}_{6} \mathrm{H}_{5} \mathrm{CH}_{2} \mathrm{O}\right), 77.1$ (d, C-2), 72.9, 71.6, and $69.6(2$ $\mathrm{PhCH} \mathrm{CH}_{2} \mathrm{O}$ and $\left.\mathrm{C}-1\right), 62.9(\mathrm{~d}, \mathrm{C}-3), 45.3\left[\mathrm{~N}\left(\mathrm{CH}_{2} \mathrm{CH}_{3}\right)_{3}\right], 8.2\left[\mathrm{~N}\left(\mathrm{CH}_{2} \mathrm{CH}_{3}\right)_{3}\right] ;{ }^{2} J_{\mathrm{C} . \mathrm{P}} 4.5,{ }^{3} J_{\text {C.P }}$ $7.0 \mathrm{~Hz} ;{ }^{1} \mathrm{H}, \delta 7.367-7.244(\mathrm{~m}, 10 \mathrm{H}, 2 \mathrm{Ph}), 6.868(\mathrm{~d}, 1 \mathrm{H}, \mathrm{PH}), 4.725$ and 4.669 (2 d, each 1 $\mathrm{H}, \mathrm{PhCH}_{2} \mathrm{O}$ ), 4.307 (s, $2 \mathrm{H}, \mathrm{PhCH}_{2} \mathrm{O}$ ), 4.071 (dq, $\left.1 \mathrm{H}, \mathrm{H}-3 \mathrm{a}\right), 4.010$ (dq, $1 \mathrm{H}, \mathrm{H}-3 \mathrm{~b}$ ), 3.825 (q, 1 H, H-2), 3.663 (dd, I H, H-la), 3.611 (dd, 1 H, H-lb), 3.045 [q, $6 \mathrm{H}$, $\left.\mathrm{N}\left(\mathrm{CH}_{2} \mathrm{CH}_{3}\right)_{3}\right], 1.326\left[\mathrm{t}, 9 \mathrm{H}, \mathrm{N}\left(\mathrm{CH}_{2} \mathrm{CH}_{3}\right)_{3}\right] ;{ }^{1} J_{\mathrm{H}, \mathrm{P}} 632 \mathrm{~Hz} ;{ }^{31} \mathrm{P}, \delta 5.5\left(\mathrm{dt},{ }^{1} J_{\mathrm{P}, \mathrm{H}} 640,{ }^{3} J_{\mathrm{P}, \mathrm{H}} 8\right.$ $\mathrm{Hz}$ ).

Methyl 2,4-di-O-benzyl-3-O-[2,3,6-tri-O-benzyl-4-O-(2,4,6-tri-O-acetyl- $\beta$-D-galactopyranosyl)- $\alpha$-D-glucopyranosyl]- $\alpha$-L-rhamnopyranoside $\quad 3^{\prime \prime}-(1,2-d i$-O-benzyl-snglycer-3-yl phosphonate) (25). - Pyridine $(2 \times 5 \mathrm{~mL})$ was evaporated from a mixture of $22(31 \mathrm{mg}, 29 \mu \mathrm{mol})$ and $24(44 \mathrm{mg}, 0.10 \mathrm{mmol})$, and the residue was dissolved in pyridine ( $2 \mathrm{~mL}$ ). Pivaloyl chloride ( $18 \mu \mathrm{L}, 0.14 \mathrm{mmol}$ ) was added, and the mixture was stirred for $2 \mathrm{~h}$, when t.l.c. $\left(9: 1 \mathrm{CH}_{2} \mathrm{Cl}_{2}-\right.$ acetone $)$ revealed the formation of $25\left(R_{\mathrm{r}} 0.63\right)$. The mixture was diluted with $\mathrm{CH}_{2} \mathrm{Cl}_{2}(50 \mathrm{~mL})$, washed with $\mathrm{M}$ triethylammonium hydrogencarbonate $(2 \times 10 \mathrm{~mL})$, dried $\left(\mathrm{Na}_{2} \mathrm{SO}_{4}\right)$, filtered, and concentrated. Column chromatography of the residue afforded 25 , isolated as a syrup $(30 \mathrm{mg}, 74 \%)$. ${ }^{1} \mathrm{H}-\mathrm{N}$.m.r. data $\left(\mathrm{CDCl}_{3}\right): \delta$ $6.853\left(\mathrm{~d}, 0.5 \mathrm{H}, J_{\mathrm{H}, \mathrm{P}} 715 \mathrm{~Hz}, \mathrm{PH}\right)$ and $6.701\left(\mathrm{~d}, 0.5 \mathrm{H}, J_{\mathrm{H}, \mathrm{P}} 726 \mathrm{~Hz}, \mathrm{PH}\right)$ of two enantiomers.

Methyl 2,4-di-O-benzyl-3-O-/2,3,6-tri-O-benzyl-4-O-(2,4,6-tri-O-acetyl- $\beta$-D-galactopyranosyl)- $\alpha$-D-glucopyranosyl $-\alpha$-L-rhamnopyranoside $\quad 3^{\prime \prime}-(1,2-d i$-O-benzyl-snglycer-3-yl triethylammonium phosphate) (26). - To compound 25 (30 mg, $21 \mu \mathrm{mol})$ was added a $0.2 \mathrm{M}$ solution of iodine in $9: 1: 1$ tetrahydrofuran-pyridine-water $(1.5 \mathrm{~mL})$. The mixture was stirred for $3 \mathrm{~h}$, when t.l.c. $\left(9: 1 \mathrm{CH}_{2} \mathrm{Cl}_{2}\right.$-acetone) indicated the absence of $\mathbf{2 5}$ and the formation of $\mathbf{2 6}\left(R_{\mathrm{F}} 0\right)$. The excess of iodine was destroyed with aq. $5 \%$ sodium hydrogensulfite, and the mixture was washed with $\mathrm{M}$ triethylammonium hydrogencarbonate $(2 \times 10 \mathrm{~mL})$, dried $\left(\mathrm{Na}_{2} \mathrm{SO}_{4}\right)$, filtered, and concentrated. Column chromatography (9:1 $\mathrm{CH}_{2} \mathrm{Cl}_{2}$-acetone followed by $\left.9: 1 \mathrm{CH}_{2} \mathrm{Cl}_{2}-\mathrm{MeOH}\right)$ of the residue gave 26, isolated as a syrup ( $30 \mathrm{mg}, 92 \%)$. N.m.r. data $\left(\mathrm{CDCl}_{3}\right):{ }^{\prime} \mathrm{H}, \delta 7.357-7.201(\mathrm{~m}, 35 \mathrm{H}, 7$ $\mathrm{Ph}$ ), 5.469 (d, $\left.1 \mathrm{HI}, \mathrm{H}-4^{\prime \prime}\right), 5.152$ (d, $\left.1 \mathrm{HI}, \mathrm{H}^{-1}{ }^{\prime}\right), 3.256$ (OMe), 2.906 [q, $\left.6 \mathrm{H}, \mathrm{N}\left(\mathrm{CH}_{2} \mathrm{CH}_{3}\right)_{3}\right]$, $1.988,1.925$, and $1.897(3 \mathrm{~s}$, each $3 \mathrm{H}, 3 \mathrm{Ac}), 1.184\left[\mathrm{t}, 9 \mathrm{H}, \mathrm{N}\left(\mathrm{CH}_{2} \mathrm{CH}_{3}\right)_{3}\right] ; J_{1^{\prime}, 2^{\prime}} 3.5, J_{3^{\prime \prime}, 4^{\prime \prime}}$ $3.1, J_{4^{\prime \prime} .5^{\prime \prime}}<1 \mathrm{~Hz} ;{ }^{31} \mathrm{P}, \delta-0.28$. 
Methyl 3-O-(4-O- $\beta$-D-galactopyranosyl- $\alpha$-D-glucopyranosyl)- $\alpha$-L-rhamnopyranoside $3^{\prime \prime}$-(sn-glycer-3-yl sodium phosphate) (2). - To a solution of $26(30 \mathrm{mg}, 20 \mu \mathrm{mol})$ in $\mathrm{CH}_{2} \mathrm{Cl}_{2}$ (1 mL) was added methanolic $7 \mathrm{M}$ ammonia $(4 \mathrm{~mL})$. After 3 days, t.l.c. (9:1 $\mathrm{CH}_{2} \mathrm{Cl}_{2}-\mathrm{MeOH}$ ) showed the deacetylation to be complete, and the mixture was concentrated. Column chromatography of the residue afforded deacetylated 26 , isolated as a syrup, $R_{\mathrm{F}} 0.57$. A solution of the residue in 2-propanol $(3 \mathrm{~mL})$ and methanol $(2 \mathrm{~mL})$, containing $10 \% \mathrm{Pd} / \mathrm{C}\left(15 \mathrm{mg}\right.$ ), was hydrogenolysed for $65 \mathrm{~h}$ at $4 \mathrm{~kg} / \mathrm{cm}^{2}$, filtered, and concentrated. Column chromatography (2:1:1 1-butanol-EtOH-water) of $50 \%$ of the residue afforded 2 , isolated as a white powder $(2.1 \mathrm{mg}, 3 \mu \mathrm{mol}) .{ }^{13} \mathrm{C}-\mathrm{N} . \mathrm{m} . \mathrm{r}$. data $\left(\mathrm{D}_{2} \mathrm{O}\right): \delta$ 103.9 and $101.8\left(\mathrm{C}-1^{\prime}, 1^{\prime \prime}\right), 96.8(\mathrm{C}-1), 67.8\left(\mathrm{~d}, \mathrm{C}-3^{\prime \prime \prime},{ }^{2} J_{\mathrm{C} P} 6.1 \mathrm{~Hz}\right), 63.4\left(\mathrm{C}-1^{\prime \prime \prime}\right), 62.3$ and

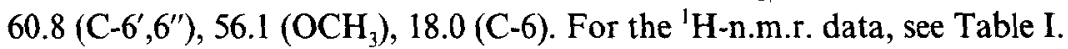

Methyl 3-O-allyl-4,6-O-benzylidene- $\beta$-D-galactopyranoside (28). - To a solution of methyl 3-O-allyl- $\beta$-D-galactopyranoside ${ }^{20}(7.60 \mathrm{~g}, 32.49 \mathrm{mmol})$ in $N, N$-dimethylformamide $(10 \mathrm{~mL})$ were added $\alpha, \alpha$-dimethoxytoluene $(25 \mathrm{~mL})$ and $p$-toluenesulfonic acid (100 mg). After $30 \mathrm{~min}$, when t.l.c. $\left(9: 1 \mathrm{CH}_{2} \mathrm{Cl}_{2}-\mathrm{EtOAc}\right)$ showed the formation of $28\left(R_{\mathrm{r}}\right.$ $0.13)$ to be complete, solid sodium hydrogencarbonate was added, and the mixture was diluted with $\mathrm{CH}_{2} \mathrm{Cl}_{2}$, washed with water, dried $\left(\mathrm{Na}_{2} \mathrm{SO}_{4}\right)$, filtered, and concentrated. Crystallisation from EtOH gave $28(7.32 \mathrm{~g}, 70 \%)$, m.p. $181^{\circ},[\alpha]_{\mathrm{p}}+54^{\circ}\left(\mathrm{c} 1, \mathrm{CHCl}_{3}\right)$. N.m.r. data $\left(\mathrm{CDCl}_{3}\right):{ }^{13} \mathrm{C}, \delta 137.6$ and $128.7-126.2\left(\mathrm{C}_{6} \mathrm{H}_{5} \mathrm{CH}\right), 134.6\left(\mathrm{H}_{2} \mathrm{C}=\mathrm{CHCH}_{2} \mathrm{O}\right)$, $117.5\left(\mathrm{H}_{2} \mathrm{C}=\mathrm{CHCH}_{2} \mathrm{O}\right), 103.7(\mathrm{C}-1), 100.8(\mathrm{PhCH}), 78.8,72.8,69.7$, and 66.4 $(\mathrm{C}-2,3,4,5), 70.4\left(\mathrm{H}_{2} \mathrm{C}=\mathrm{CHCH}_{2} \mathrm{O}\right), 69.1(\mathrm{C}-6), 56.7\left(\mathrm{OCH}_{3}\right){ }^{1} \mathrm{H}, \delta 7.531-7.325(\mathrm{~m}, 5 \mathrm{H}$, $\mathrm{Ph}), 5.974\left(\mathrm{~m}, 1 \mathrm{H}, \mathrm{H}_{2} \mathrm{C}=\mathrm{CHCH}_{2} \mathrm{O}\right), 5.545(\mathrm{~s}, 1 \mathrm{H}, \mathrm{PhCH}), 5.330$ and $5.219(2 \mathrm{~m}$, each 1 $\mathrm{H}, \mathrm{H}_{2} \mathrm{C}=\mathrm{CHCH}_{2} \mathrm{O}$ ), 4.357 (dd, $\left.1 \mathrm{H}, \mathrm{H}-6 \mathrm{a}\right), 4.267$ (d, $\left.1 \mathrm{H}, \mathrm{H}-1\right), 4.090$ (dd, $\left.1 \mathrm{H}, \mathrm{H}-6 \mathrm{~b}\right)$, 3.953 (dd, $1 \mathrm{H}, \mathrm{H}-2), 3.587$ (s, $3 \mathrm{H}, \mathrm{OMe}$ ), 3.475 (dd, $1 \mathrm{H}, \mathrm{H}-3), 3.431$ (m, $1 \mathrm{H}, \mathrm{H}-5) ; J_{1,2}$ $7.8, J_{2,3} 9.7, J_{3,4} 3.5, J_{4,5}<1, J_{5,6 \mathrm{a}} 1.6, J_{5,6 \mathrm{~b}} 1.9, J_{6 \mathrm{a}, 6 \mathrm{~b}}-12.4 \mathrm{~Hz}$.

Anal. Calc. for $\mathrm{C}_{17} \mathrm{H}_{22} \mathrm{O}: \mathrm{C}, 63.34 ; \mathrm{H}, 6.88$. Found: C, 63.15; H, 7.15.

Methyl 3-O-allyl-4,6-O-benzylidene-2-O-(2,3,4,6-tetra-O-benzyl- $\alpha, \beta$-D-glucopyranosyl)- $\beta$-D-galactopyranoside $(29 \alpha \beta)$. - To a stirred solution of ethyl 2,3,4,6-tetra- $O$ benzyl-1-thio- $\beta$-D-glucopyranoside ${ }^{19}(27 ; 1.54 \mathrm{~g}, 4.80 \mathrm{mmol}), 28(2.50 \mathrm{~g}, 4.24 \mathrm{mmol})$, and powdered molecular sieves $(4 \AA, 10 \mathrm{~g})$ in dry ether $(100 \mathrm{~mL})$ was added methyl triflate $(2.3 \mathrm{~mL}, 21.2 \mathrm{mmol})$. After $18 \mathrm{~h}$, t.l.c. (25:2 toluene-acetone) indicated the absence of $28\left(R_{\mathrm{F}} 0.03\right)$ and the presence of $29 \alpha\left(R_{\mathrm{F}} 0.23\right)$ and $29 \beta\left(R_{\mathrm{F}} 0.15\right)$. Triethylamine $(9 \mathrm{~mL})$ was added, stirring was continued for $10 \mathrm{~min}$, and the mixture was filtered through Celite and concentrated. Column chromatography $\left(97: 3 \mathrm{CH}_{2} \mathrm{Cl}_{2}-\mathrm{EtOAc}\right)$ of the residue yielded $29 \alpha(2.02 \mathrm{~g}, 56 \%)$, m.p. $127^{\circ}$ (from EtOH), $[\alpha]_{\mathrm{p}}+97^{\circ}\left(c 1, \mathrm{CHCl}_{3}\right)$, followed by $29 \beta\left(1.20 \mathrm{~g}, 33 \%\right.$ ), m.p. $162^{\circ}$ (from EtOH), $[\alpha]_{\mathrm{p}}+42^{\circ}\left(\mathrm{c} 1, \mathrm{CHCl}_{3}\right)$. N.m.r. data $\left(\mathrm{CDCl}_{3}\right): 29 \alpha{ }^{13} \mathrm{C}, \delta 138.9-135.5$ and $128.7-126.4\left(\mathrm{C}_{6} \mathrm{H}_{5} \mathrm{CH}_{2} \mathrm{O}\right.$ and $\left.\mathrm{C}_{6} \mathrm{H}_{5} \mathrm{CH}\right), 134.7$ $\left(\mathrm{H}_{2} \mathrm{C}=\mathrm{CHCH}_{2} \mathrm{O}\right), 117.5\left(\mathrm{H}_{2} \mathrm{C}=\mathrm{CHCH}_{2} \mathrm{O}\right), 104.5(\mathrm{C}-1), 101.1(\mathrm{PhCH}), 95.7\left(\mathrm{C}-1^{\prime}\right)$, 81.9, 79.5, 78.2, 77.7, 73.1, 72.3, 69.8, and $66.3\left(\mathrm{C}-2,3,4,5,2^{\prime}, 3^{\prime}, 4^{\prime}, 5^{\prime}\right), 75.4,74.4,69.7$, 72.2, 70.8, 69.1, and 68.4 (4 $\mathrm{PhCH}_{2} \mathrm{O}, \mathrm{H}_{2} \mathrm{C}=\mathrm{CHCH}_{2} \mathrm{O}$, and C-6,6'), $56.4\left(\mathrm{OCH}_{3}\right) ;{ }^{1} \mathrm{H}, \delta$ 7.534-7.118(m, 25 H, 5 Ph), 5.914(m, $\left.1 \mathrm{H}, \mathrm{H}_{2} \mathrm{C}=\mathrm{CHCH}_{2} \mathrm{O}\right), 5.579\left(\mathrm{~d}, 1 \mathrm{H}, \mathrm{H}-1^{\prime}\right), 5.534$ (s, $1 \mathrm{H}, \mathrm{PhCH}$ ), 5.247 and $5.084\left(2 \mathrm{~m}\right.$, each $\left.\mathrm{I} \mathrm{H}, \mathrm{H}_{2} \mathrm{C}=\mathrm{CHCH}_{2} \mathrm{O}\right), 4.967,4.832,4.800$, $4.787,4.703,4.619,4.498$, and 4.932 (8 d, each $\left.1 \mathrm{H}, 4 \mathrm{PhCH}_{2} \mathrm{O}\right), 4.483(\mathrm{~d}, 1 \mathrm{H}, \mathrm{H}-1)$, 
4.240 (d, $1 \mathrm{H}, \mathrm{H}-4), 3.532$ (s, $3 \mathrm{H}, \mathrm{OMe}$ ), 3.386 (bs, $1 \mathrm{H}, \mathrm{H}-5) ; J_{1,2} 7.8, J_{3,4} 3.6, J_{4,5}<1, J_{1^{\prime}, 2}$ $3.7 \mathrm{~Hz} ; 29 \beta{ }^{13} \mathrm{C}, \delta 138.7-136.0$ and $128.8-127.3\left(C_{6} \mathrm{H}_{5} \mathrm{CH}_{2} \mathrm{O}\right.$ and $\left.\mathrm{C}_{6} \mathrm{H}_{5} \mathrm{CH}\right), 134.9$ $\left(\mathrm{H}_{2} \mathrm{C}=\mathrm{CHCH}_{2} \mathrm{O}\right), 117.2\left(\mathrm{H}_{2} \mathrm{C}=\mathrm{CHCH}_{2} \mathrm{O}\right), 102.5(2 \mathrm{C})$ and $101.3\left(\mathrm{C}-1,1^{\prime}\right.$ and $\left.\mathrm{PhCH}\right)$, $55.5\left(\mathrm{OCH}_{3}\right) ;{ }^{\prime} \mathrm{H}, \delta 7.161-7.533(\mathrm{~m}, 25 \mathrm{H}, 5 \mathrm{Ph}), 5.854\left(\mathrm{~m}, \mathrm{l} \mathrm{H}, \mathrm{H}_{2} \mathrm{C}=\mathrm{CHCH}_{2} \mathrm{O}\right), 5.515$ (s, $1 \mathrm{H}, \mathrm{PhCH}), 5.174$ and $5.046\left(2 \mathrm{~m}\right.$, each $\left.1 \mathrm{H}, \mathrm{H}_{2} \mathrm{C}=\mathrm{CHCH}_{2} \mathrm{O}\right), 5.046,4.913,4.807$, 4.784, 4.727, 4.644, 4.561, and 4.542 ( $8 \mathrm{~d}$, each $1 \mathrm{H}, 4 \mathrm{PhCH}_{2} \mathrm{O}$ ), 4.917 (d, I H, H-1'), 4.422 (d, $1 \mathrm{H}, \mathrm{H}-1$ ), 3.521 (s, $3 \mathrm{H}, \mathrm{OMe}$ ), 3.378 (bs, $1 \mathrm{H}, \mathrm{H}-5$ ); $J_{1,2} 7.7, J_{1^{\prime}, 2^{\prime}} 8.0 \mathrm{~Hz}$.

Anal. Calc. for $\mathrm{C}_{51} \mathrm{H}_{56} \mathrm{O}_{11}: \mathrm{C}, 72.49 ; \mathrm{H}, 6.68$. Found $29 \alpha: \mathrm{C}, 72.57 ; \mathrm{H}, 6.65$. Found 29ß: C, 72.45; H, 6.76 .

Methyl 3-O-allyl-6-O-benzyl-2-O-(2,3,4,6-tetra-O-benzyl- $\alpha$-D-glucopyranosyl)$\beta$-D-galactopyranoside (30). - A solution of $29 \alpha(2.02 \mathrm{~g}, 2.39 \mathrm{mmol})$, borane-trimethylamine complex ( $1.05 \mathrm{~g}, 14.39 \mathrm{mmol})$, and powdered molecular sieves $(4 \AA, 5.0 \mathrm{~g})$ in tetrahydrofuran $^{7}(50 \mathrm{~mL})$ was stirred for $1 \mathrm{~h}$. Aluminium(III) chloride $(1.89 \mathrm{~g}, 14.17$ mmol) was added at $0^{\circ}$ and the mixture was stirred for $16 \mathrm{~h}$ at room temperature. T.l.c. $\left(95: 5 \mathrm{CH}_{2} \mathrm{Cl}_{2}-\mathrm{EtOAC}\right)$ then showed the conversion of $29 \propto\left(R_{\mathrm{F}} 0.62\right)$ into $30\left(R_{\mathrm{F}} 0.47\right)$. The mixture was diluted with $\mathrm{CH}_{2} \mathrm{Cl}_{2}(400 \mathrm{~mL})$, filtered through Celite, washed with $\mathrm{M}$ $\mathrm{H}_{2} \mathrm{SO}_{4}(3 \times 50 \mathrm{~mL})$, water, aq. $5 \%$ sodium hydrogencarbonate, and water, dried $\left(\mathrm{Na}_{2} \mathrm{SO}_{4}\right)$, filtered, and concentrated. Column chromatography of the residue yielded 30, isolated as a syrup $(1.54 \mathrm{~g}, 76 \%),[x]_{\mathrm{n}}+51^{\circ}\left(\mathrm{cl}, \mathrm{CHCl}_{3}\right)$. N.m.r. data $\left(\mathrm{CDCl}_{3}\right):{ }^{13} \mathrm{C}, \delta$ $138.8-137.3$ and $128.2-127.3\left(\mathrm{C}_{6} \mathrm{H}_{5} \mathrm{CH}_{2} \mathrm{O}\right), 133.9\left(\mathrm{H}_{2} \mathrm{C}=\mathrm{CHCH}_{2} \mathrm{O}\right), 118.3\left(\mathrm{H}_{2} \mathrm{C}=\right.$ $\left.\mathrm{CHCH}_{2} \mathrm{O}\right), 104.4(\mathrm{C}-1), 95.7\left(\mathrm{C}-1^{\prime}\right), 75.5,74.5,73.7,73.3,72.2,71.0,69.0$, and $68.4(5$ $\mathrm{PhCH}_{2} \mathrm{O}, \mathrm{H}_{2} \mathrm{C}=\mathrm{CHCH}_{2} \mathrm{O}$, and $\left.\mathrm{C}-6,6^{\prime}\right), 56.4\left(\mathrm{OCH}_{3}\right) ;{ }^{1} \mathrm{H}, \delta 7.358-7.136(\mathrm{~m}, 25 \mathrm{H}, 5 \mathrm{Ph})$, $5.889\left(\mathrm{~m}, 1 \mathrm{H}, \mathrm{H}_{2} \mathrm{C}=\mathrm{CHCH}_{2} \mathrm{O}\right), 5.542\left(\mathrm{~d}, 1 \mathrm{H}, \mathrm{H}-\mathrm{l}^{\prime}\right), 5.220$ and $5.094(2 \mathrm{~m}$, each $1 \mathrm{H}$, $\left.\mathrm{H}_{2} \mathrm{C}=\mathrm{CHCH}_{2} \mathrm{O}\right), 4.411(\mathrm{~d}, 1 \mathrm{H}, \mathrm{H}-1), 3.505$ (s, $\left.3 \mathrm{H}, \mathrm{OMe}\right), 3.478(\mathrm{dd}, 1 \mathrm{H}, \mathrm{H}-3) ; J_{1,2} 7.9$, $J_{2.3} 9.6, J_{3,4} 3.4, J_{1: 2}, 3.7 \mathrm{~Hz}$.

Anal. Calc. for $\mathrm{C}_{51} \mathrm{H}_{58} \mathrm{O}_{11}: \mathrm{C}, 72.32 ; \mathrm{H}, 6.90$. Found: $\mathrm{C}, 72.24 ; \mathrm{H}, 7.07$.

Methyl 3-O-allyl-6-O-benzyl-4-O-(2,3,4,6-tetra-O-acetyl- $\beta$-D-glucopyranosyl)-2O-(2,3,4,6-tetra-O-benzyl- $\alpha$-D-glucopyranosyl)- $\beta$-D-galactopyranoside (32). To a stirred mixture of $30(1.54 \mathrm{~g}, 1.81 \mathrm{mmol}), 2,3,4,6$-tetra- $O$-acetyl- $\alpha$-D-glucopyranosyl trichloroacetimidate $(31 ; 1.49 \mathrm{~g}, 3.02 \mathrm{mmol})$, and powdered molecular sieves $(4 \AA, 1.0 \mathrm{~g})$ in dry $\mathrm{CH}_{2} \mathrm{Cl}_{2}(40 \mathrm{~mL})$ was added trimethylsilyl triflate $(250 \mu \mathrm{L}, 1.38 \mathrm{mmol})$ at $-30^{\circ}$. The temperature was gradually raised to $-10^{\circ}$ and, when t.1.c. [7:3 light petroleum (b.p. 40-60 $)$-EtOAc] showed the absence of $30\left(R_{\mathrm{F}} 0.31\right)$ and a new u.v.-positive product (32, $R_{\mathrm{F}} 0.18$ ), pyridine ( $2 \mathrm{~mL}$ ) was added, and the mixture was filtered through Celite and concentrated. Column chromatography [6:4 light petroleum (b.p. 40-60 )-EtOAc] of the residue afforded 32 , isolated as a syrup $(1.92 \mathrm{~g}, 90 \%),[\alpha]_{\mathrm{o}}+34^{\circ}\left(\mathrm{c} 1, \mathrm{CHCl}_{3}\right)$. N.m.r. data $\left(\mathrm{CDCl}_{3}\right):{ }^{13} \mathrm{C}, \delta 170.4169 .3\left(\mathrm{COCH}_{3}\right), 138.8-138.0$ and $128.3-127.3\left(\mathrm{C}_{6} \mathrm{H}_{5} \mathrm{CH}_{2} \mathrm{O}\right)$, $133.9\left(\mathrm{H}_{2} \mathrm{C}=\mathrm{CHCH}_{2} \mathrm{O}\right), 117.6\left(\mathrm{H}_{2} \mathrm{C}=\mathrm{CHCH}_{2} \mathrm{O}\right), 104.4(\mathrm{C}-1), 99.3\left(\mathrm{C}-1^{\prime \prime}\right), 96.0\left(\mathrm{C}-1^{\prime}\right)$, $56.3\left(\mathrm{OCH}_{3}\right), 20.6\left(\mathrm{COCH}_{3}\right) ;{ }^{1} \mathrm{H}, \delta 7.365-7.126(\mathrm{~m}, 25 \mathrm{H}, 5 \mathrm{Ph}), 5.879(\mathrm{~m}, 1 \mathrm{H}$, $\left.\mathrm{H}_{2} \mathrm{C}=\mathrm{CHCH}_{2} \mathrm{O}\right), 5.501\left(\mathrm{~d}, 1 \mathrm{H}, \mathrm{H}-1^{\prime}\right), 3.459(\mathrm{OMe}), 2.037,2.030,2.022$, and $2.008(4 \mathrm{~s}$, each $3 \mathrm{H}, 4 \mathrm{Ac}) ; J_{1^{\prime}, z^{\prime}} 3.7 \mathrm{~Hz}$.

Anal. Calc. for $\mathrm{C}_{65} \mathrm{H}_{76} \mathrm{O}_{20}: \mathrm{C}, 66.31 ; \mathrm{H}, 6.51$. Found: C, 65.85; $\mathrm{H}, 6.51$.

Methyl 6-O-benzyl-4-O-(2,3,4,6-tetra-O-acetyl- $\beta$-D-glucopyranosyl)-2-0-(2,3,4, 
6-tetra-O-benzyl- $\alpha$-D-glucopyranosyl)- $\beta$-D-galactopyranoside (33). - To a solution of $32(0.75 \mathrm{~g}, 0.49 \mathrm{mmol})$ in 7:3:1 EtOH-toluene--water $(10 \mathrm{~mL})$ was added tris(triphenylphosphine)rhodium(I) chloride ${ }^{21}$ (95 $\mathrm{mg}, 0.1 \mathrm{mmol}$ ). The mixture was boiled under reflux for $30 \mathrm{~h}$ (t.l.c., 9:1 $\mathrm{CH}_{2} \mathrm{Cl}_{2}-$ EtOAc, $33 R_{\mathrm{F}}$ 0.26), then concentrated. Column chromatography (85:15 $\left.\mathrm{CH}_{2} \mathrm{Cl}_{2}-\mathrm{EtOAc}\right)$ of the residue yielded 33 , isolated as a glass $(0.27 \mathrm{~g}, 49 \%),[\alpha]_{\mathrm{D}}+28^{\circ}\left(c 0.9, \mathrm{CHCl}_{3}\right)$. N.m.r. data $\left(\mathrm{CDCl}_{3}\right):{ }^{13} \mathrm{C}, \delta 170.3-169.2$ $\left(\mathrm{COCH}_{3}\right), 138.5-137.5$ and $128.2-127.3\left(\mathrm{C}_{6} \mathrm{H}_{5} \mathrm{CH}_{2} \mathrm{O}\right), 103.1(\mathrm{C}-1), 100.7\left(\mathrm{C}-1^{\prime \prime}\right), 97.5(\mathrm{C}-$ $\left.1^{\prime}\right), 56.6\left(\mathrm{OCH}_{3}\right), 20.4\left(\mathrm{COCH}_{3}\right) ;{ }^{1} \mathrm{H}, \delta 7.385-7.092(\mathrm{~m}, 25 \mathrm{H}, 5 \mathrm{Ph}), 5.198\left(\mathrm{~d}, 1 \mathrm{H}, \mathrm{H}-1^{\prime}\right)$, 4.331 (d, $1 \mathrm{H}, \mathrm{H}-1$ ), 3.422 (s, $3 \mathrm{H}, \mathrm{OMe}$ ), 2.043, 2.018, and 2.001 ( $3 \mathrm{~s}, 3,6,3 \mathrm{H}, 4 \mathrm{Ac}) ; J_{1,2}$ $7.6, J_{1^{\prime}, 2^{\prime}} 3.9 \mathrm{~Hz}$.

Anal. Calc. for $\mathrm{C}_{62} \mathrm{H}_{70} \mathrm{O}_{20}: \mathrm{C}, 65.60 ; \mathrm{H}, 6.21$. Found: C, 65.39; $\mathrm{H}, 6.47$.

Methyl 2-O- $\alpha$-D-glucopyranosyl-4-O- $\beta$-D-glucopyranosyl- $\beta$-D-galactopyranoside (3). - To a solution of $33(58.4 \mathrm{mg}, 51.4 \mu \mathrm{mol})$ in $\mathrm{MeOH}(4 \mathrm{~mL})$ was added sodium methoxide to $\mathrm{pH} 10$. The mixture was stirred for $16 \mathrm{~h}$, neutralised with Dowex $-50\left(\mathrm{H}^{+}\right)$ resin, filtered, and concentrated. The residue was taken up in $\mathrm{EtOH}(9 \mathrm{~mL})$ and EtOAc $(1 \mathrm{~mL})$, and $10 \% \mathrm{Pd} / \mathrm{C}(25 \mathrm{mg})$ was added. Hydrogenolysis was performed for $16 \mathrm{~h}$ at 4 $\mathrm{kg} / \mathrm{cm}^{2}$, and the solution was filtered, concentrated, and lyophilised to afford 3 , isolated as a white powder $(25 \mathrm{mg}, 95 \%),[\alpha]_{\mathrm{D}}+65^{\circ}\left(c 1, \mathrm{H}_{2} \mathrm{O}\right)$. N.m.r. data $\left(\mathrm{D}_{2} \mathrm{O}\right):{ }^{13} \mathrm{C}, \delta 105.5$ and $105.2\left(\mathrm{C}-1,1^{\prime \prime}\right), 99.7\left(\mathrm{C}-1^{\prime}\right), 79.7,77.9,77.2,77.1,75.4,75.0,74.1,73.2,72.9,72.7$, 70.9 , and $70.6\left(\mathrm{C}-2,3,4,5,2^{\prime}, 3^{\prime}, 4^{\prime}, 5^{\prime}, 2^{\prime \prime}, 3^{\prime \prime}, 4^{\prime \prime}, 5^{\prime \prime}\right), 62.0(2 \mathrm{C}), 61.5\left(\mathrm{C}-6,6^{\prime}, 6^{\prime \prime}\right), 58.6\left(\mathrm{OCH}_{3}\right)$. For the 'H-n.m.r. data, see Table I.

Methyl 6-O-benzyl-4-O-(2,3,4,6-tetra-O-acetyl- $\beta$-D-glucopyranosyl)-2-O- $(2,3,4$, 6-tetra-O-benzyl- $\alpha$-D-glucopyranosyl)- $\beta$-D-galactopyranoside 3-(1,2-di-O-benzyl-snglycer-3-yl phosphonate) (34). - Pyridine $(2 \times 5 \mathrm{~mL})$ was evaporated from a mixture of 33 (112 mg, $99 \mu \mathrm{mol})$ and 24 (66 mg, $150 \mu \mathrm{mol}$ ), and the residue was dissolved in dry pyridine $(2 \mathrm{~mL})$. Pivaloyl chloride $(46 \mu \mathrm{L}, 375 \mu \mathrm{mol})$ was added and the mixture was stirred for $2 \mathrm{~h}$, when t.l.c. $\left(8: 2 \mathrm{CH}_{2} \mathrm{Cl}_{2}\right.$ EtO $\left.\Lambda \mathrm{c}\right)$ showed that $33\left(R_{\mathrm{F}} 0.51\right)$ had been converted almost completely into $34\left(R_{\mathrm{F}} 0.41\right)$. Water was added, and the solution was concentrated, diluted with $\mathrm{CH}_{2} \mathrm{Cl}_{2}(100 \mathrm{~mL})$, washed with $\mathrm{M}$ triethylammonium hydrogencarbonate $(2 \times 25 \mathrm{~mL})$, dried $\left(\mathrm{Na}_{2} \mathrm{SO}_{4}\right)$, filtered, and concentrated. Column chromatography of the residue yielded the two enantiomers of 34 , isolated as a syrup (100 mg, 70\%). N.m.r. data $\left(\mathrm{CDCl}_{3}\right)$ : ${ }^{1} \mathrm{H}, \delta 6.959\left(\mathrm{~d}, 0.5 \mathrm{H}, J_{\mathrm{H}, \mathrm{P}} 707 \mathrm{~Hz}, \mathrm{PH}\right), 6.901(\mathrm{~d}, 0.5 \mathrm{H}$, $\left.J_{\mathrm{H}, \mathrm{P}} 726 \mathrm{~Hz}, \mathrm{PH}\right) ;{ }^{31} \mathrm{P}, \delta 11.10\left(\mathrm{dq},{ }^{1} J_{\mathrm{P}, \mathrm{H}} 707,{ }^{3} J_{\mathrm{P}, \mathrm{H}} 10 \mathrm{~Hz}\right)$ and $8.87\left(\mathrm{dq},{ }^{1} J_{\mathrm{P}, \mathrm{H}} 726,{ }^{3} J_{\mathrm{P}, \mathrm{H}} 10\right.$ $\mathrm{Hz}$ ).

Methyl 6-O-benzyl-4-O-(2,3,4,6-tetra-O-acetyl- $\beta$-D-glucopyranosyl)-2-O-(2,3,4, 6-tetra-O-benzyl- $\alpha$-D-glucopyranosyl)- $\beta$-D-galactopyranoside $3-(1,2-d i-0$-benzyl-snglycer-3-yl triethylammonium phosphate) (35). - To a solution of $34(80 \mathrm{mg}, 55 \mu \mathrm{mol})$ in tetrahydrofuran $(2 \mathrm{~mL})$ was added $0.35 \mathrm{M}$ iodine in $1: 1$ pyridine-water $(700 \mu \mathrm{L})$. After 1 h, t.l.c. (8:2 $\left.\mathrm{CH}_{2} \mathrm{Cl}_{2}-\mathrm{EtOAc}\right)$ indicated complete formation of $35\left(R_{\mathrm{F}} 0\right)$, and aq. $10 \%$ sodium hydrogensulfite was added to destroy the excess of iodine. The mixture was diluted with $\mathrm{CH}_{2} \mathrm{Cl}_{2}(100 \mathrm{~mL})$, washed with $\mathrm{M}$ triethylammonium hydrogencarbonate (2 $\times 20 \mathrm{~mL})$, dried $\left(\mathrm{Na}_{2} \mathrm{SO}_{4}\right)$, filtered, and concentrated. Column chromatography $(2: 1$ $\mathrm{CH}_{2} \mathrm{Cl}_{2}-\mathrm{MeOH}$ ) of the residue on $\mathrm{LH}-20$ gave 35 , isolated as a syrup ( $86 \mathrm{mg}, 99 \%$ ), $[\alpha]_{\mathrm{D}}$ $+36^{\circ}\left(c 1, \mathrm{CHCl}_{3}\right)$. N.m.r. data $\left(\mathrm{CDCl}_{3}\right):{ }^{13} \mathrm{C}, \delta 170.5-169.3\left(4 \mathrm{COCH}_{3}\right), 138.8-138.2$ 
and 128.1-127.3 $\left(\mathrm{C}_{6} \mathrm{H}_{5} \mathrm{CH}_{2} \mathrm{O}\right), 103.8(\mathrm{C}-1), 100.0\left(\mathrm{C}-1^{\prime \prime}\right), 96.9\left(\mathrm{C}-1^{\prime}\right), 65.3(\mathrm{~d}, \mathrm{C}-3$ glycerol, $\left.{ }^{2} J_{\mathrm{C} . \mathrm{P}} 6.3 \mathrm{~Hz}\right), 56.1\left(\mathrm{OCH}_{3}\right), 44.9\left[\mathrm{~N}\left(\mathrm{CH}_{2} \mathrm{CH}_{3}\right)_{3}\right], 20.8-20.4\left(\mathrm{COCH}_{3}\right), 8.2$ $\left[\mathrm{N}\left(\mathrm{CH}_{2} \mathrm{CH}_{3}\right)_{3}\right] ;{ }^{1} \mathrm{H}, \delta$ 7.348-7.114(m, 35 H, $\left.7 \mathrm{Ph}\right), 5.437\left(\mathrm{~d}, 1 \mathrm{H}, \mathrm{H}-1^{\prime}\right), 5.379(\mathrm{~d}, 1 \mathrm{H}, \mathrm{H}-$ $\left.1^{\prime \prime}\right), 4.271$ (d, $\left.1 \mathrm{H}, \mathrm{H}-1\right), 3.379$ (s, $\left.3 \mathrm{H}, \mathrm{OMe}\right), 2.649$ [q, $6 \mathrm{H}, \mathrm{N}\left(\mathrm{CH}_{2} \mathrm{CH}_{3}\right)_{3}$ ], 2.124, 2.005. 1.996 , and $1.954(4 \mathrm{~s}$, each $3 \mathrm{H}, 4 \mathrm{Ac}), 1.008\left[\mathrm{t}, 9 \mathrm{H}, \mathrm{N}\left(\mathrm{CH}_{2} \mathrm{CH}_{3}\right)_{3}\right] ; J_{1,2} 7.7, J_{1,2}, 3.8, J_{1^{\prime \prime 2}, 2^{\prime \prime}}$ $8.1 \mathrm{~Hz} ;{ }^{3 !} \mathrm{P}, \delta-0.51$.

Methyl 2-O- $\alpha$-D-glucopyranosyl-4-O- $\beta$-D-glucopyranosyl- $\beta$-D-galactopyranoside 3-( sn-glycer-3-yl sodium phosphate) (4). - To a solution of 35 ( $59 \mathrm{mg}, 38 \mu \mathrm{mol}$ ) in 1:1 $\mathrm{CH}_{2} \mathrm{Cl}_{2}-\mathrm{MeOH}(2 \mathrm{~mL})$ was added methanolic $7 \mathrm{M}$ ammonia $(3 \mathrm{~mL})$. After $48 \mathrm{~h}$, the solvent was evaporated, and the residue was purified by column chromatography $(2: 1$ $\mathrm{CH}_{2} \mathrm{Cl}_{2}-\mathrm{MeOH}$ ) on Sephadex $\mathrm{LH}-20$ to yield deacetylated 35, isolated as a syrup (40 $\mathrm{mg}, 80 \%$ ). An aliquot (33 mg, $25 \mu \mathrm{mol}$ ) of this product was dissolved in 2-propanol ( 3 $\mathrm{mL})$, and $\mathrm{MeOH}(2 \mathrm{~mL})$ and $10 \% \mathrm{Pd} / \mathrm{C}(25 \mathrm{mg})$ were added. Hydrogenolysis was performed for $16 \mathrm{~h}$ at $4 \mathrm{~kg} / \mathrm{cm}^{2}$, the mixture was filtered through Celite and concentrated, and a solution of the residue in water was treated with Dowex-50 $\left(\mathrm{Na}^{+}\right)$resin. Lyophilisation of the filtrate afforded 4 , isolated as a white powder $(17.7 \mathrm{mg}, 98 \%),[\alpha]_{\mathrm{D}}$ $+40^{\circ}\left(c 1, \mathrm{H}_{2} \mathrm{O}\right)$. N.m.r data $\left(\mathrm{D}_{2} \mathrm{O}\right):{ }^{13} \mathrm{C}, \delta 105.7$ and $104.0\left(\mathrm{C}-1,1^{\prime \prime}\right), 99.1\left(\mathrm{C}-\mathrm{l}^{\prime}\right), 67.7(\mathrm{~d}$, C- $\left.3^{\prime \prime \prime},{ }^{2} J_{\mathrm{C} . \mathrm{P}} 5.2 \mathrm{~Hz}\right), 63.5\left(\mathrm{C}-1^{\prime \prime \prime}\right), 72.1\left(\mathrm{~d}, \mathrm{C}-2^{\prime \prime \prime},{ }^{3} J_{\mathrm{C} . \mathrm{P}} 7.7 \mathrm{~Hz}\right), 62.1,61.8$, and 61.7 $\left(\mathrm{C}-6,6^{\prime}, 6^{\prime \prime}\right), 58.6\left(\mathrm{OCH}_{3}\right) ;{ }^{31} \mathrm{P}, \delta 3.10$. For the ${ }^{1} \mathrm{H}-\mathrm{n} . \mathrm{m} . \mathrm{f}$. data, see Table I.

\section{ACKNOWLEDGMENTS}

This investigation was supported by the Netherlands Foundation for Chemical Research (SON/NWO), the Institute of Molecular Biology and Medical Biotechnology (IMB, Utrecht University), and the Netherlands Innovation Directed Programme for Biotechnology (IOP-b). We thank Drs. P. van Seeventer for his help during synthesis, and Drs. K. Hård, J. P. M. Lommerse, C. H. Hokke, and P. de Waard for recording the 2D-n.m.r. spectra.

\section{REFERENCES}

1 J. E. G. van Dam, A. Fleer, and H. Snippe, Antonie van Leeuwenhoek: J. Microbiol. Serol. , 58 (1990) $1-47$.

2 C. Lugowski and H. J. Jennings, Carbohydr. Res., 131 (1984) 119-129.

3 J. B. Robbins, R. Austrian, C.-J. Lee, S. C. Rastogi, G. Schiffman, J. Henrichsen, P. H. Mäkelä, C. V. Broome, R. R. Facklam, R. H. Tiesjema, and J. C. Parke, I. Infect. Dis., 148 (1983) 1136 -1159.

4 A. Lipták, P. Fügedi, and P. Nánási, Carbohydr. Res., 65 (1978) 209-217.

5 A. F. Bochkov and A. Ch. Dzhein, Bull. Acad. Sci. U.S.S.R., (1968) 168169.

6 M. E. Evans, Carbohydr. Res., 21 (1972) 168169.

7 M. Ek, P. J. Garegg, H. Hultberg, and S. Oscarson, J. Carbohydr. Chem., 2 (1983) 473475.

8 R. R. Schmidt and M. Stumpp, Liebigs Ann. Chem., (1983) $1249-1256$.

9 P. Kovac, C. P. J. Glaudemans, and R. B. Taylor, Carbohydr. Res, 142 (1985) $158 \cdots 164$.

10 B. Classon, P. J. Garegg, and B. Samuelsson, Acta. Chem. Scand, Ser. B, 38 (1984) 419-422.

11 A. M. P. van Steijn, J. P. Kamerling, and J. F. G. Vliegenthart, Carbohydr. Res., 211 (1991) 261277.

12 H. Lönn, J. Carbohydr. Chem., 6 (1987) 301-306.

13 H. Paulsen and M. Paal, Carbohydr. Res., 135 (1984) 53-69.

14 S. Sato, Y. Ito, T. Nakada, Y. Nakahara, and T. Ogawa, Carbohydr. Res., 167 (1987) 197 210

15 D. Marion and K. Wüthrich, Biochem. Biophys. Res. Commun., 113 (1983) 967-974. 
16 A. Bax and D. G. Davis, J. Magn. Reson., 65 (1985) 355-360.

17 C. A. A. van Boeckel, Thesis, Leiden University, The Netherlands, 1982.

18 E. de Vroom, C. E. Dreef, H. van den Elst, G. A. van der Marel, and J. H. van Boom, Recl. Trav. Chim. Pays-Bas, 107 (1988) 592-595.

19 F. Weygand and H. Ziemann, Justus Liebigs Ann. Chem, (1962) 179-198.

20 K. Kohato, S. A. Abbas, and K. L. Matta, Carbohydr. Res., 132 (1984) 127-135.

21 E. J. Corey and W. J. Suggs, J. Org. Chem., 38 (1973) 3224. 